\title{
Review of Fluorescence-Based Velocimetry Techniques to Study High-Speed Compressible Flows (Invited)
}

\author{
Brett F. Bathel ${ }^{*}$ \\ NASA Langley Research Center, Hampton, Virginia, 23681 \\ Craig Johansen ${ }^{\dagger}$ \\ University of Calgary, Calgary, Alberta, Canada, T2N 1N4 \\ Jennifer A. Inman ${ }^{\ddagger}$, Stephen B. Jones ${ }^{\S}$, and Paul M. Danehy ${ }^{* *}$ \\ NASA Langley Research Center, Hampton, Virginia, 23681
}

\begin{abstract}
This paper reviews five laser-induced fluorescence-based velocimetry techniques that have been used to study high-speed compressible flows at NASA Langley Research Center. The techniques discussed in this paper include nitric oxide (NO) molecular tagging velocimetry (MTV), nitrogen dioxide photodissociation $\left(\mathrm{NO}_{2}\right.$-to-NO) MTV, and $\mathrm{NO}$ and atomic oxygen (O-atom) Doppler-shift-based velocimetry. Measurements of both singlecomponent and two-component velocity have been performed using these techniques. This paper details the specific application and experiment for which each technique has been used, the facility in which the experiment was performed, the experimental setup, sample results, and a discussion of the lessons learned from each experiment.
\end{abstract}

\section{Nomenclature}

$\begin{array}{ll}\text { AoA } & =\text { angle-of-attack } \\ c & =\text { speed of light, } \mathrm{m} / \mathrm{s} \\ C C D & =\text { charge coupled device } \\ C F D & =\text { computational fluid dynamics } \\ L I F & =\text { laser-induced fluorescence } \\ \lambda & =\text { wavelength, nm } \\ M T V & =\text { molecular tagging velocimetry } \\ N O & =\text { nitric oxide } \\ N O_{2} & =\text { nitrogen dioxide } \\ P_{\text {static }} & =\text { static pressure, Pa } \\ P L I F & =\text { planar laser-induced fluorescence } \\ P M T & =\text { photomultiplier tube } \\ S L P M & =\text { standard liters per minute } \\ S N R & =\text { signal-to-noise ratio } \\ T_{\text {static }} & =\text { static temperature, } \mathrm{K} \\ t_{\text {add }} & =\text { additional delay, ns } \\ t_{E 1} & =\text { first exposure duration, } \mathrm{ns} \\ t_{E 2} & =\text { second exposure duration, } \mathrm{ns} \\ t_{G D} & =\text { gate delay, ns } \\ t_{G W} & =\text { intensifier gate width, } \mathrm{ns}\end{array}$

\footnotetext{
${ }^{*}$ Graduate Student, University of Virginia and NASA Graduate Co-op, Hampton, Virginia, AIAA Student Member.

${ }^{\dagger}$ Assistant Professor, Mechanical and Manufacturing Engineering, AIAA Member.

${ }^{\sharp}$ Research Scientist, Advanced Sensing and Optical Measurement Branch, MS 493, AIAA Member.

${ }^{\S}$ Research Technician, Advanced Sensing and Optical Measurement Branch, MS 493.

${ }^{k * *}$ Research Scientist, Advanced Sensing and Optical Measurement Branch, MS 493, AIAA Associate Fellow.
} 


$\begin{array}{ll}t_{P} & =\text { probe laser duration, } \mathrm{ns} \\ \Delta t_{D 2} & =\text { second exposure delay, } \mathrm{ns} \\ \Delta t_{P D} & =\text { probe laser delay, } \mathrm{ns} \\ \Delta t_{P U M P} & =\text { pump laser delay, } \mathrm{ns} \\ \tau_{L I F} & =\text { fluorescence lifetime, } \mathrm{ns} \\ x & =\text { streamwise spatial coordinate, } \mathrm{m} \\ U & =\text { streamwise component of velocity, } \mathrm{m} / \mathrm{s} \\ V & =\text { radial component of velocity, } \mathrm{m} / \mathrm{s} \\ v & =\text { wave number, } \mathrm{cm}^{-1}\end{array}$

\section{Introduction}

$\mathrm{T}$ HE design of hypersonic aerospace vehicles requires an understanding of the gas flow passing over a vehicle at Mach numbers on the order of 5 or higher. The interactions that can occur between this flowfield and the vehicle have a substantial influence on the vehicle and subsystem designs. These subsystems include the thermal protection system and flight control systems, among many others. Computational fluid dynamic (CFD) simulation capabilities can potentially predict the behavior of these corresponding flowfields and the underlying physical mechanisms which govern them. However, these simulation tools often rely on models that make certain thermal, chemical, and/or mathematical assumptions about the flowfield. The complexity of a hypersonic flow makes it difficult to determine which simplifying assumptions are valid. Consequently, such simulation tools require experimental validation to ensure that the predicted behavior matches the true physical flowfield behavior. Experiments can also often uncover flow phenomena that are not present or readily apparent in computational studies.

The validation of CFD tools also requires an understanding of the test environment in which the hypersonic flow experiments are performed. These test facilities often have operational uncertainties which can affect experimental results. For instance, the flow composition, chemistry, and flow steadiness may be unknown or not well understood. If the assumed facility flow behavior suffers from uncertainties or is inaccurate, the assumed boundary conditions used in the corresponding CFD simulation will result in a predicted flowfield that may differ significantly from experimental observations, even if the selected computational methodology otherwise captures all of the significant flow physics. Therefore, experiments are additionally needed to characterize the flowfields produced in hypersonic test facilities.

To obtain quantitative data, the measurement techniques used in hypersonic flowfield measurements must also be properly calibrated and the measurement uncertainties must be well characterized. Efforts to reduce measurement uncertainty should also be made so that analysis of spatially and temporally sensitive flow phenomena can be performed and be compared with CFD solutions. Consideration should also be given to the type of measurement technique used in a particular application. For hypersonic flowfield measurements, the use of physical probes can introduce unwanted flow structures or perturbations into the flow, such as shock waves and unsteadiness, which can affect the particular property being measured.

In this paper, work on the implementation and application of five velocity measurement techniques using laserinduced fluorescence (LIF) at NASA Langley Research Center is discussed. The techniques include 1) single-laser nitric oxide (NO) molecular tagging velocimetry (MTV) using a single-frame camera; 2) single-laser NO MTV using a double-frame camera; 3 ) three-laser nitrogen dioxide $\left(\mathrm{NO}_{2}\right)$ photolysis and subsequent NO MTV using a double-frame camera; 4) single-laser, two-photon excitation of oxygen atom (O-atom) Doppler-based fluorescence velocimetry and 5) a Doppler-based NO fluorescence velocimetry. The work has been motivated by many of the factors already described including fundamental gas flowfield studies, hypersonic vehicle CFD validation, facility characterization, and the development and implementation of quantitative, non-intrusive hypersonic flowfield measurement techniques in NASA research facilities.

Section II contains a discussion of the five techniques listed above. For each technique, the motivation for using the particular velocimetry method is discussed. Descriptions of each experimental setup and facility in which the technique has been applied are included. For each technique, a discussion of the uncertainties associated with the measurement is provided along with sample experimental results obtained by application of the respective technique. Finally, a discussion of the lessons learned while conducting each experiment and suggested improvements for each technique is given. 


\section{Discussion of Fluorescence-Based Velocimetry Techniques}

\section{A. Single-Laser NO MTV with a Single-Frame Camera}

Molecular-tagging velocimetry works by selectively marking (i.e. tagging) some molecules in a flow, taking two pictures of those molecules with a known time separation, and calculating the velocity by measuring the displacement of the tagged molecules in the second image relative to their position in the first. The molecules (or atoms, in the case of certain tracer species) are tagged by tuning a monochromatic light source (a laser) to a frequency that is resonant with an electronic transition in the tracer species. The excited molecules may fluoresce (i.e. relax to a lower energy state radiatively, by emitting a photon) or may dissipate the energy non-radiatively through collisions. It is the fluorescence process that allows the location of the tracer molecules to be captured by an imaging detector. The advantages of nitric oxide as the tracer species are that it has a molecular weight comparable to air or nitrogen (so it can be expected to follow the flow without suffering from particle lag or buoyancy effects) and that it is usually chemically stable (so it can be stored in a pressurized botle at room temperate prior to being seeded into a flow). It also can be generated by photolysis of $\mathrm{NO}_{2}$, as described in section $\mathrm{C}$ below. Its primary disadvantage is its toxicity and the fact that it must be seeded into most flows since it is not naturally present in many facilities. When a single laser pulse is used to excite NO, the maximum time separation between the two velocimetry images is limited by the fact that fluorescence exhibits an exponential decay as a function of time.

In the experiments described in this section, the monochromatic light source consisted of a three-part laser system. A frequency-doubled Nd:YAG laser $(532 \mathrm{~nm}$ ) pumped a pulsed dye laser (Spectra Physics Quanta Ray PDL-2) to generate yellow light (near $574 \mathrm{~nm}$ ). This yellow light was frequency-doubled and then mixed with residual infrared light from the Nd:YAG fundamental $(1064 \mathrm{~nm})$ in a wavelength extender (Spectra-Physics Quanta Ray WEX-1C), resulting in tunable light near $226 \mathrm{~nm}$. Fluorescence was imaged using a 16 bit PIMax-II intensified CCD camera, which acquires single-frame images at a rate of $10 \mathrm{~Hz}$, synchronized with, but delayed from the laser by $\sim 500 \mathrm{~ns}$.

A series of one-dimensional velocity distributions in a flow can be obtained using this technique. Repeated measurements can be used to acquire statistical quantities such as average and fluctuating velocities in the flow. A single laser is used to excite multiple lines of $\mathrm{NO}$ and the displacement of these lines is imaged using a single-frame camera to compute velocity. One advantage of this technique is the reduction in the camera and laser equipment requirements compared to other methods detailed below. Another is that the delayed gate can have the intensifier gain increased arbitrarily relative to the first gate (since the two images are acquired independently, at different times). This can result in a better signal to noise ratio than the double-frame camera technique (described below) in which a single gain setting must be chosen for both the first and second images.

Figure 1 shows a generalized schematic of the laser pathway. To form a laser sheet, the collimated $226 \mathrm{~nm}$ probe beam is passed through a cylindrical lens, which expands the beam in one direction while leaving it collimated in the other. A spherical lens (typically 0.5 or 1 meter focal length) then collimates the diverging axis of the beam and focuses the other axis into a thin sheet approximately 0.5 -mm thick.

To tag multiple lines of $\mathrm{NO}$ in the test section for the velocimetry measurement described in this section, a spatial comb filter was used. This was placed below the sheet-forming optics and was used to create a series of vertical shadows. The tines of the spatial comb filter were approximately $1-\mathrm{mm}$ wide with a spacing of 2-mm and the resulting lines had a minimum thickness of about $0.5 \mathrm{~mm}$. This technique was applied to air flows without naturally occurring nitric oxide. Mass flow controllers were used to control the relative flow rates of nitrogen and nitric oxide, generally resulting in a seeding-gas mixture of $99.5 \% \mathrm{~N}_{2}$ to $0.5 \% \mathrm{NO}$. This mixture was found to give adequate signal with minimal absorption of the laser sheet over the range of conditions tested. A series of underexpanded jet tests were conducted using the 15-Inch Mach 6 test section used as a vacuum chamber. The tests were designed to determine the conditions and mechanisms governing the transition from laminar to turbulent behavior in both impinging and free (i.e. non-impinging) underexpanded jet flows. Detailed results and analysis of

American Institute of Aeronautics and Astronautics 
these tests can be found in Refs. 1, 2, and 3. A stainless steel plenum wrapped in electrical heater tape and insulation was placed in the test section and supplied a heated, premixed, pressurized source of gas to either a sonic (converging) or supersonic (converging-diverging) nozzle. The ambient pressure in the test chamber ranged from $0.12 \mathrm{kPa}$ to $9.63 \mathrm{kPa}$, and the plenum pressure ranged from 0.87 $\mathrm{kPa}$ to $136.54 \mathrm{kPa}$, resulting in nozzle-exit to ambient pressure ratios of up to 37.6. The plenum temperature ranged from $400 \mathrm{~K}$ to $600 \mathrm{~K}$. Firing of the laser flash lamps, laser Q-switch and the CCD intensifier gate were coordinated using trigger pulses generated by a LabSmith LC880 Programmable Experiment Controller (Trigger ${ }^{\mathrm{TM}}$ software, version 5.02).

To measure velocity, two images are needed: a reference image and a time-delayed image. The reference image can be acquired with the camera gate opening just prior to the laser pulse and capturing an image before the fluorescing gas has convected downstream. This reference image can also be taken when the flow velocity is negligible, such as in the stagnant test chamber after it has been allowed to fill with the seeded gas mixture. The time-delayed image is then acquired after increasing the timing delay between laser pulse and camera gate to allow the gas to convect downstream some finite amount. At some test conditions, we found that there was enough residual seeded gas mixture in the test chamber (and far enough away from the jet flow) to serve as a self-referencing mechanism in single-shot delayed images, obviating the need for a separate "zero-delay" reference image.

Image processing entailed smoothing the images with a spatial filter to reduce random noise, as wel as subtracting a background image. Then, in the reference image (or in the reference regions outside the flow), a straight line was fit to each tagged line which then served as the zero-delay reference. In the

delayed image (or in the non-reference regions of the flow), line centers were found by fitting a parabola to the measured intensity. Figure 2 shows an example of the data (thick lines) and the fitted reference lines (thin lines) for a case where self-referencing was used.
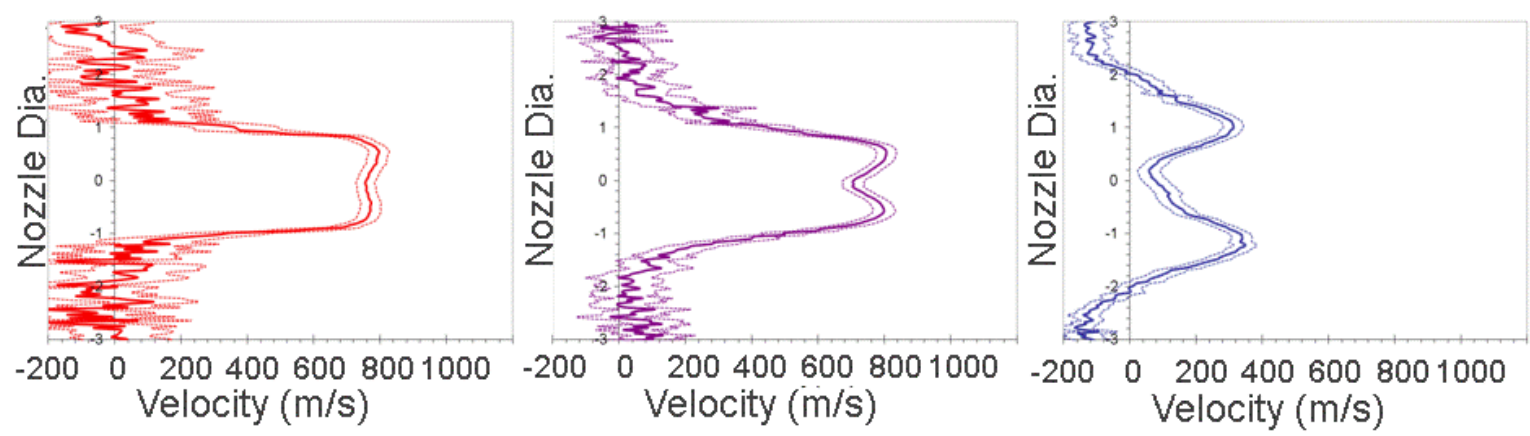

Figure 4. Velocity Profiles with Error Bars. These profiles correspond to Lines 1, 11, and 22 in Fig. 3. Dashed lines represent the upper and lower 95\% confidence intervals. (Taken from Ref. 1, Fig. 8).

4

American Institute of Aeronautics and Astronautics 
In order to calculate velocity from this measured displacement, the effective delay after the laser pulse, $\Delta t$, is needed. The effective delay time is that time at which half of the total fluorescence would have been acquired. If the fluorescence lifetime $\tau_{L I F}$ were effectively constant during the exposure time (i.e. intensifier gate width, $t_{G W}=$ $200 \mathrm{~ns}$ ) - then $\Delta t$ would simply be the gate delay $t_{G D}$ plus half the gate width, $t_{G W}$. However, because $\tau_{L I F}$ is on the order of the exposure time, more fluorescence is acquired near the beginning of the exposure than at the end. As a result, the effective time delay is less than $\left(t_{G D}+1 / 2 t_{G W}\right)$. By modeling the fluorescence intensity as an exponential decay and integrating the intensity from the time the intensifier gate opens, $t_{G D}$, to the time it closes, $t_{G D}+t_{G W}$, we get the total acquired intensity in arbitrary units.

The time at which half the total intensity has been acquired is a function of gate delay and local fluorescence lifetime. Fluorescence lifetimes of between $157 \mathrm{~ns}$ and $188 \mathrm{~ns}$ were expected for the range of conditions encountered in the underexpanded jet tests for all but the first (leftmost) tagged line. The runs presented in this paper used a gate delay of $t_{G D}=750 \mathrm{~ns}$, resulting in a $\Delta t$ of $822.5+/-2.5 \mathrm{~ns}$. (For the first line, the fluorescence lifetime was estimated to get as low as $140 \mathrm{~ns}$. In order to account for this wider range, we increased the uncertainty in the additional delay, $t_{\text {add }}$, to $+2.5,-5.5 \mathrm{~ns}$, in order to account for the wider range of possible fluorescence lifetimes in this portion of the flow.)

An additional correction factor related to the finite fluorescence lifetime, the finite intensifier gate width, and the fact that fluorescing molecules with a non-zero velocity move during the exposure time was included in velocity calculations. This is described briefly in the following section and Ref. 5 gives expressions for calculating this correction factor for different gas compositions, fluorescence lifetimes, and gate widths. Examples of the results obtained in these underexpanded jet studies are presented in Figs. 3-6 for cases with a Mach 2.6 nozzle where the jet was impinging upon a target. Figure 3 shows full-field velocity profiles for a selected case where the impingement target was located 15.2 nozzle diameters downstream of the nozzle exit. In this figure, the displacement of the thick line from the thin reference line is proportional to the velocity. Error bars are shown as dashed lines on either side of each thick line. Figure 4 shows three of these profiles in greater detail. Plots of the measured centerline velocity are given in Fig. 5 for this case and in Fig. 6 for a case where the impingement target was closer (10.7 nozzle diameters) to the nozzle exit. Note the presence of a large recirculation region, as evidenced by the negative centerline velocities, for the case in Fig. 6.

A potentially large source of error was neglected in our analysis. This error arises in regions where there is a left-to-right gradient in the amount of quenching across the width of a measurement line. Quenching is primarily a function of pressure. Therefore, if strong pressure gradients exist between the left and right edges of a measurement line, significant errors could result. ${ }^{6}$ The two places in underexpanded jet flows where this is likely to occur are near the nozzle exit (that is, along the first measurement line within the jet) and across normal shock waves. Other competing effects, like the increase in density across a shock, may lead to almost constant signal intensity across the shock, reducing the effect of this error. Suffice it to say that caution should be exercised in interpreting measurements along a normal shock wave, if for no other reason than that strong velocity gradients exist within such a measurement volume.

The largest contribution to the overall uncertainty of these measurements was the random error associated with measuring the shifted fluorescence profiles. Increasing the fluorescence signal, the magnification, and/or the number of single-shot images (for mean velocity measurements) would reduce uncertainty. In addition, the algorithm for identifying poor fits to the data was rudimentary and has since been improved. The effects of quenching gradients in the flow should be included in the uncertainty analysis of flows where strong quenching gradients could exist across a single measurement line. Decreasing the gate delay could have improved the measurement precision. A gate delay of between 300 and $500 \mathrm{~ns}$ is recommended as a way to increase the average image intensity, especially if the magnification of the imaging system can be increased so that sufficiently large pixel shifts are still observed. For this particular type of jet flow, modifications to the test apparatus and testing procedure could be made with the intention of more effectively trapping NO in the zero-velocity region of the flow. Any such modification which helped to increase the signal-to-noise ratio throughout the image (but especially in regions far from the jet) would help to reduce measurement error. For example, an F/2 lens compared to the F/4.5 lens used in this current work would have collected four times more LIF signal. ${ }^{4}$ A custom long-pass filter (from Layertec, $\mathrm{GmbH}$ ) has since been used in place of the UG-5 filter, which allows additional fluorescence signal to be collected while still blocking scattered laser light. For arrangements using a supposedly static region as a zero-velocity reference, measurements should be made or CFD performed at the conditions of the tests to ascertain the validity of the assumption of negligible velocity in the reference regions. Finally, Doppler velocimetry could be used to measure the spanwise component of velocity and thus extend the current methodology to a two-dimensional velocimetry technique as described in section II.D.2. 


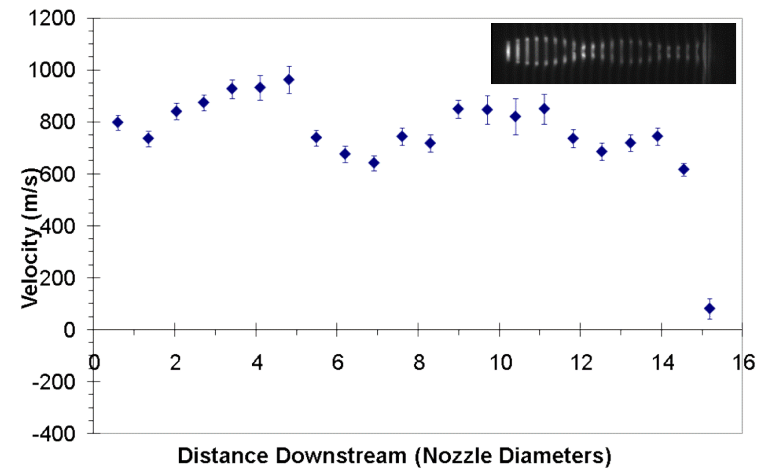

Figure 5. Centerline Velocity. Same case as in Figs. 2-4. Error bars mark upper and lower $95 \%$ confidence intervals. Impingement target was 15.2 nozzle diameters from the nozzle exit. (Taken from Ref. 1, Fig. 9)

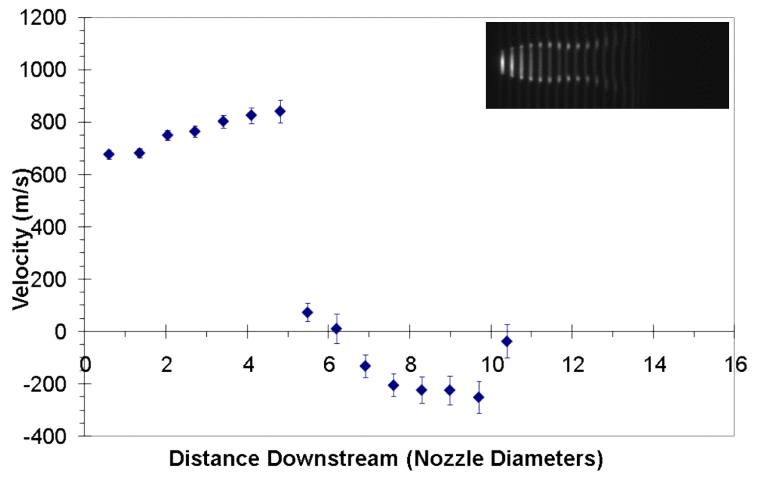

Figure 6. Centerline Velocity. This case has a larger pressure ratio and a shorter impingement distance (10.7 nozzle diameters) than the case in Fig. 5. (Taken from Ref. 1, Fig. 11)

In summary, measured velocities ranged from approximately -200 to $+1,000 \mathrm{~m} / \mathrm{s}$ with accuracies on the order of 15 to $50 \mathrm{~m} / \mathrm{s}$ with the higher accuracies in regions of higher signal-to-noise. These accuracies, however, rely upon the assumption of zero average velocity in the region between approximately 3.5 and 5.3 nozzle diameters away from the jet centerline. Any deviation from zero average velocity in the reference region will lead to a direct error of the same amount in the measured velocity. This velocimetry technique was shown to have the additional advantage of providing partial flow-visualization since there are a large number of relatively closely-spaced lines through the flowfield. Flow visualization can further the understanding of features in velocity profiles by, for example, showing the location and shape of shock waves and expansion regions. The single-image technique reported herein theoretically eliminates the limitation of similar two-image techniques which restricts them to measurements in steady flows (or to averaged measurements, with potential systematic biases, in unsteady flows). However, for reliable single-shot measurements of velocity fluctuations in unsteady flows, higher signal-to-noise images are needed.

\section{B. Single-Laser NO MTV with a Double-Frame Camera}

Improvement of the single-excitation NO MTV technique was motivated by the need for quantitative flowfield measurement capabilties in NASA hypersonic test facilities. For example, quantitative measurements of laminar and laminar-unsteady hypersonic boundary layer behavior, ${ }^{5-7}$ reaction control system (RCS) jet behavior in wake flows, ${ }^{5,6,8,9}$ and facility characterization ${ }^{10}$ were of particular interest. Since these types of experiments were to be made in flows with both a steady and unsteady nature, the quantitative measurements to be used required the ability to probe the flowfield with relatively high spatial and temporal accuracy and precision. The relatively large mechanical vibrations associated with hypersonic facility operation could result in increased experimental uncertainty in instances when the single-frame single-camera system, the profile-forming optics, or the laser system may be displaced as a result of such vibrations. A similar concern exists for a single-frame, two-camera system. Therefore, a decision was made to use a double-framing camera that could provide temporally correlated image pairs to visualize the displacement of the MTV profiles.

The camera selected was a 12-bit Cooke DiCAM-PRO, utilizing an intensified 1280 by 1024 pixel array interline Figure 7. Schematic of laser alignment for single-laser NO progressive scan CCD. For most of the experiments, a much MTV flat plate experiments. (Adapted from Ref. 18, Fig. 2) 
smaller vertical region of the CCD array was typically used to allow for a read-out rate of $10 \mathrm{~Hz}$, corresponding to the laser pulse frequency. When used in double frame mode, the camera can acquire an image pair with a minimum $\Delta t_{D 2}=500 \mathrm{~ns}$ delay. This delay is between the end of the first gate and the beginning of the second and is a hardware limitation. Each gate has a minimum duration of $20 \mathrm{~ns}$, with delay settings and durations controllable in increments of $20 \mathrm{~ns}$. The gain setting for the zero-delay and delayed frames cannot be adjusted independently for each frame.

The laser system was modified to include a Sirah Cobra Stretch dye laser (which replaced the PDL) that was pumped at $532 \mathrm{~nm}$ to achieve a $622 \mathrm{~nm}$ output beam. This output was mixed with $355 \mathrm{~nm}$ light from the Spectra Physics Pro $230 \mathrm{Nd}$ :YAG laser in a Sirah Frequency Conversion Unit (which replaced the WEX) to produce $\sim 5 \mathrm{~mJ}$ per pulse at $226 \mathrm{~nm}$ with a spectral line width of $\sim 0.07 \mathrm{~cm}^{-1}$. The laser pulse duration, $t_{P}$, was $\sim 9.5 \mathrm{~ns}$. The beam path used to direct the laser light into the test section was similar to that described in section II.A. and is shown in Fig. 7.

For all of the experiments described in Refs. 5-10 (with the exception of the Orion RCS jet experiment described in Refs. 5 and 6), a cylindrical lens array consisting of 251 -m focal length cylindrical lenses diffusion-welded together was used in place of the comb filter to form the individual laser lines to tag the NO molecules. The lines formed by the lens array had a separation distance of approximately $2.25-\mathrm{mm}$, widths of approximately $1.1 \mathrm{~mm}$ at FWHM (full width at half maximum) and a $0.5-\mathrm{mm}$ thickness. Several factors influenced the spatial broadening of the profiles formed by the lens array beyond the theoretical spot size of approximately $0.25-\mathrm{mm}$. These included: (1) the imaged region and focal point of the lens array did not overlap, (2) laser saturation of the spectral transition resulting in spatial broadening, and (3) various optical beam distortions.

Figure 8(a) shows a generalized timing sequence adopted for these experiments. The vertical axis represents fluorescence intensity, with the solid purple line showing the exponential decay behavior of fluorescence with time for typical run static pressures at room temperature $\left(\mathrm{P}_{\text {static }}<800 \mathrm{~Pa}, \mathrm{~T}_{\text {static }}=300 \mathrm{~K}\right)$. The light gray shaded regions are indicative of the first and second exposure durations, $t_{E I}$ and $t_{E 2}$ respectively. The light purple shaded region indicates the laser probe beam duration, $t_{p}$. The darker shaded regions represent the time integrated signal intensities captured during the first and second exposures.

Prior to performing any experiments, a timing sequence with the first exposure completely enveloping the probe laser pulse and having the shortest possible setting $\left(t_{E 1}=20 \mathrm{~ns}\right)$ and a second exposure on the order $t_{E 2}=300 \mathrm{~ns}$ was used. Originally it was thought that this timing sequence would capture sufficient signal intensities in the first and second exposures to make a velocity measurement while also being essentially flow-stopping (i.e. relatively short exposure and frame delay times relative to the characteristic flow time scale). However, when this approach was used, the signal in the first exposure proved to be much greater than that in the second. When the signal captured in the first exposure exceeded approximately $2 / 3$ of the dynamic range of the camera, artifacts from the first exposure were present on the intensifier phosphor that were observed in the second exposure, which adversely affected the velocity measurements. Since the camera's single gain setting did not allow for the gain in the first exposure to be set independent of the second, the end of the first exposure was set to coincide with the beginning of the probe laser, thereby limiting the signal collected.

The relatively long duration of the second exposure with respect to the first exposure presented another issue. While the longer duration was chosen to obtain comparable signal intensities relative to the first exposure, the highspeed motion of the tagged profiles made the measurement susceptible to motion blurring issues. The issue of motion blurring in MTV experiments had been addressed by Danehy et al. ${ }^{11}$ in which a correction to the effective time between the first and second exposures was made by assuming a constant fluorescence intensity during the respective exposures. However, analysis in Refs. 5 and 6 showed that the exponential fluorescence decay rate in a region of uniform collisional quenching $\left(\mathrm{Q}_{21}=\right.$ constant $)$ weighted the measured intensities in the direction opposite of the gas motion. The result of this additional weighting is shown in Fig. 8(b). Assuming uniform collisional quenching, a method of correcting the velocities for the effects of motion blurring and exponential fluorescence decay was developed using a phenomenological geometric argument based upon camera- and laser-timing parameters.

In regions where the collisional quenching varied spatially, the velocity measurements are susceptible to overbiasing if the quenching gradient is positive in the direction of motion and underbiasing if the quenching gradient is negative in the direction of motion. This problem was discussed in Ref. 5 which described measurements of Orion reaction control system (RCS) jet velocities that were greater than expected in the immediate vicinity of the nozzle exit.

This technique measures a single-component of velocity, namely, the component perpendicular to the tagged lines (i.e. profiles). Components of flow velocity parallel to the tagged profiles contribute to increased measurement uncertainty. In Refs. 5-7, measurements were made in the boundary layer of a flat plate, with the laser sheet parallel to (and a small distance above) the surface of the flat plate. The streamwise component of velocity was measured, 
with the profiles running spanwise. Portions of the hypersonic boundary layer had a component of velocity in the spanwise direction, parallel to the plate surface, induced by the presence of a tripping element. The uncertainty associated with this motion resulted from gas being convected in the spanwise direction between the first and second exposures. If the gas then moved in the spanwise direction between the first and second exposures, its new location in the second exposure would be incorrectly correlated with a different portion of gas in the first exposure. Reference 6 provides a methodology for estimating the magnitude of this error source. Uncertainties in the mean velocity varied between approximately $3 \%$ and $21 \%$ of the measured mean velocity for wall-normal and planview streamwise velocity measurements. Laser scatter on the wall of the flat plate model limited how closely measurements of streamwise velocity could be made to the wall. Based on analysis of the side-view velocity profiles in Ref. 7, it was estimated that the closest a measurement of streamwise velocity could be made to the plate surface was $0.38-\mathrm{mm}$.

Figures 9 and 10 show sample data from Ref.7 using this technique. Mean velocity is indicated by the center of black horizontal bars whose width corresponds to the uncertainty in the mean. In Fig. 9, mean streamwise velocity profiles obtained from a set of 106 single-shot images extending in the wall-normal direction are shown at several streamwise positions along the centerline of a flat plate model. The gray box denotes the outline of a 2-mm tall by 4$\mathrm{mm}$ diameter cylindrical trip fixed to the plate surface at approximately $75.4 \mathrm{~mm}$ downstream of the sharp leading edge.

A reversal of the flow direction extending nearly to the height of the tripping element is observed just in front of the trip $(x=7.22 \mathrm{~cm})$, indicating a large separated flow region forward of the trip. A subsequent comparison between this data set and computations ${ }^{12}$ showed relatively good agreement between the measured and predicted streamwise velocity for most of the profiles. However, unpublished comparisons between computations and measurements of the velocity immediately upstream of the tripping element showed discrepancies, with the measured velocities consistently lower than those predicted." Based upon this result, it was hypothesized that the presence of a velocity gradient would bias the velocity measurement either low or high if the flow were locally decelerating or accelerating, respectively. While further study of this issue is needed, it is suggested that if known velocity gradients exist within a flowfield in which MTV measurements are made, only a Lagrangian-type description of the tagged profiles (i.e. profile displacements and the associated time delay between image pairs) should be reported for comparison with CFD, rather than reporting velocities. Another factor that complicates this type of measurement is model displacement relative to the position of the laser lines used for the MTV measurement. Over the course of an experiment any such displacement may result in additional measurement errors.

Figure 10 shows a magnified view of Fig. 9 where three centerline mean velocity profiles were measured immediately in front of, above, and after the tripping element with respect to the streamwise direction. In this figure, the uncertainties in the mean velocity appear relatively constant up to a wall-normal position of approximately 2.0

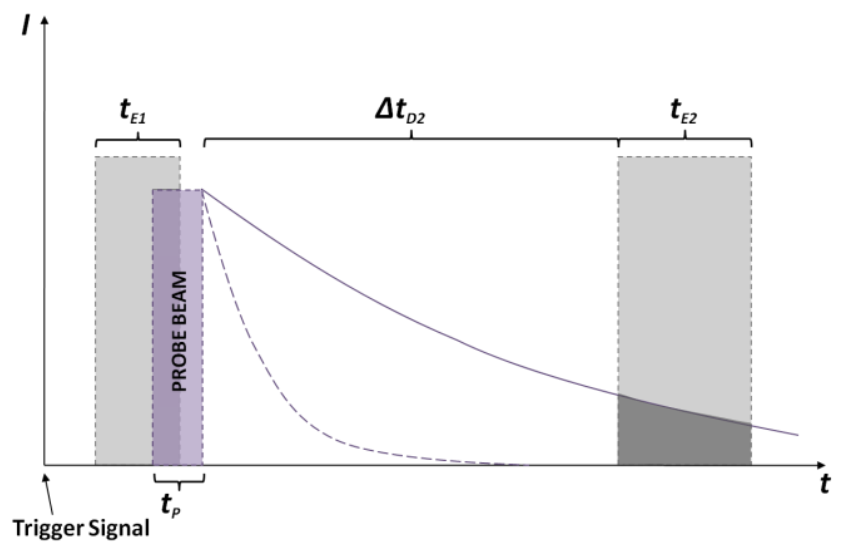

(a)

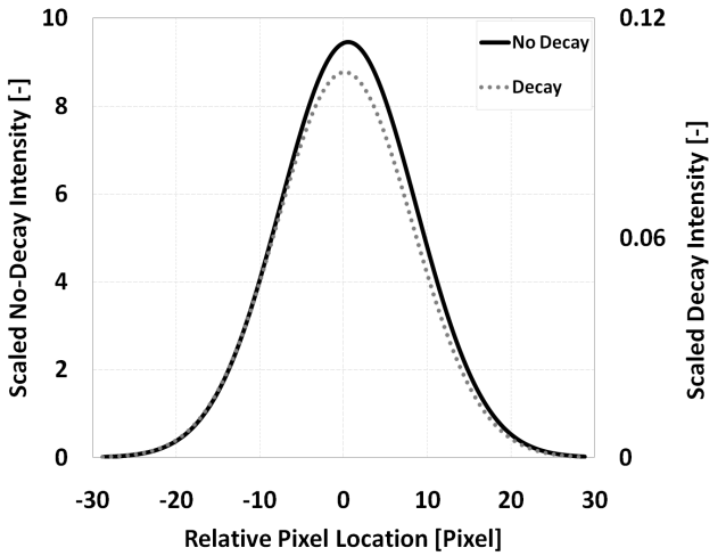

(b)

Figure 8. (a) Camera and laser timing sequence for single-laser, double-frame camera NO MTV technique. Solid line represents NO fluorescence intensity decay at moderate pressures. Dashed line represents NO fluorescence intensity decay at elevated pressures (adapted from Ref. 6, Fig. 1). (b) Simulated $2^{\text {nd }}$ gate CCD exposure using a Gaussian 19-pixel FWHM spatial laser profile (adapted from Ref. 6, Fig. 3). The fluorescence lifetime is approximately $150 \mathrm{~ns}$. The gas velocity is $500 \mathrm{~m} / \mathrm{s}$ with a magnification of $5.70 \times 10^{-2} \mathrm{~mm} /$ pixel. Solid line represents constant fluorescence intensity over the duration of the exposure while the dotted line represents a fluorescence intensity that decays exponentially with a lifetime of $150 \mathrm{~ns}$.

\footnotetext{
* Mahesh, K., University of Minnesota, February 2011 (private communication).
} 
$\mathrm{mm}$. The measurement points nearest to the edge of the boundary layer exhibit larger uncertainties in the mean caused by reduced and/or intermittent signal levels near the boundary layer edge. This is thought to be a result of either unsteadiness in the seeding and entrainment of NO gas into the boundary layer from the seeding slot, small facility-based acoustic disturbances, or some combination of the two.

This double-frame technique was also applied to an arc jet flow, as described in Ref. 10. Figure 11 shows measurements along profiles spaced $0.57,1.97$, and $2.97 \mathrm{~cm}$ downstream of the nozzle exit of the arc jet, denoted by horizontal bars. Again, the widths of these bars indicate the uncertainty in the measurement. Figure 11(a) shows the mean (filled symbols) and fluctuating component (open symbols) of axial velocity obtained from 240 single-shot images. Figure 11(b) shows a single-shot velocity measurement along the same profiles (with correspondingly higher uncertainty).

In this particular experiment, a method of relating the single-shot measurement spatial uncertainty to the signalto-noise ratio (SNR) was developed since the SNR had the largest influence on measurement uncertainty in singleshot measurements. In Fig. 11(b), the intermittent presence of NO and reduced signal levels in the core of the nozzle jet flow often resulted in single-shot velocity profiles that were sparsely populated with useable data, which increased the single-shot uncertainty. Outside of this core flow, the consistent presence of NO and strong signal intensity resulted in consistent single-shot measurements with relatively small single-shot uncertainties.

By quantifying the single-shot measurement uncertainty, a methodology to estimate the magnitude of the fluctuating axial velocity component was developed. This involved subtracting the contribution of the instrument error as a function of SNR from the measured standard deviation in mean axial velocity. The open symbols in Fig. 11(a) represent the estimated fluctuation in axial velocity using this method. One drawback of this method was that the data used to characterize the relationship between the SNR and single-shot measurement spatial uncertainty was obtained from the flowfield itself. The data were taken from a region of the flowfield outside of the nozzle jet core flow that was assumed to be essentially quiescent and assumed to have a known velocity from which information regarding the instrument error could be obtained. If small variations in velocity existed within this region, the corresponding instrument error would be overestimated. Therefore, in future experiments an effort will be made to make separate measurements for instrument error quantification.

Using this single-laser NO MTV technique with a double-frame camera, uncertainties in the mean velocity in the core of the arc jet flow were approximately $2 \%$ the measured mean velocity. Single-shot uncertainties in this region were approximately $20 \%$ of the measured single-shot velocity. Recall that the boundary layer experiments described above (and in Refs. 5-7), had uncertainties in the mean between $3 \%$ and $21 \%$. 


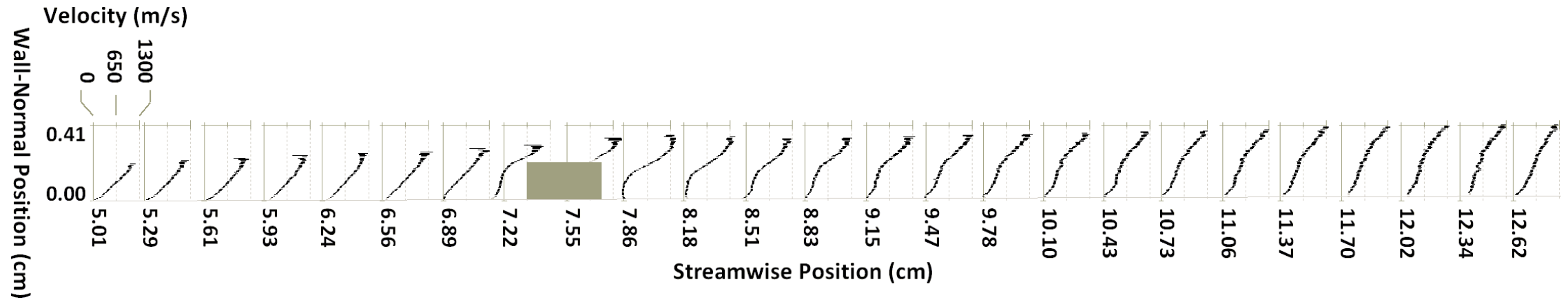

Figure 9. Centerline mean streamwise velocity measurements along flat plate with cylindrical trip for $\operatorname{Re}_{\mathrm{x}}=1.7 \mathrm{x} 10^{6} \mathrm{~m}^{-1}$. Width of bars indicates uncertainty in the measured mean values [Taken from Ref. 7, Fig. 1(c)]

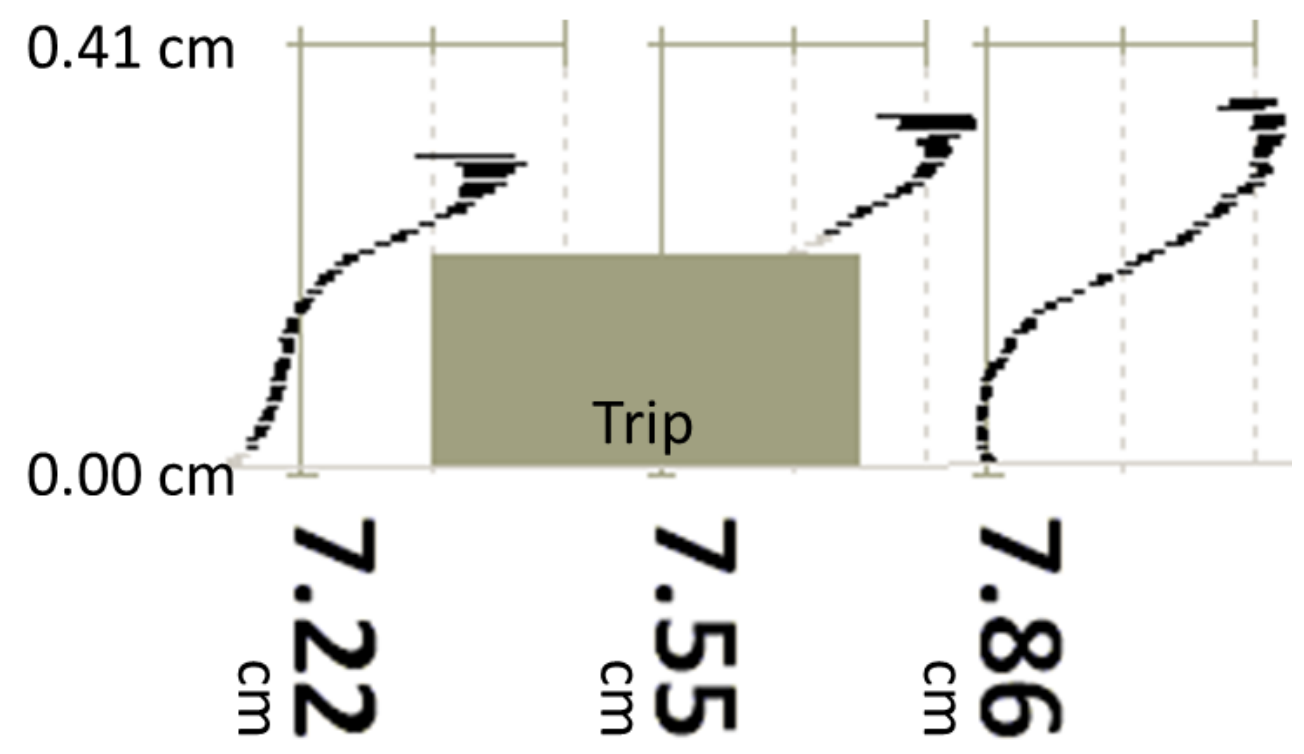

Figure 10. Centerline mean streamwise velocity measurements in the immediate vicinity of a $2.0 \mathrm{~mm}$ tall by $4.0 \mathrm{~mm}$ diameter cylindrical tripping element. This figures is a magnified view of Fig. 9. [Adapted from Ref. 7, Fig. 1(c)]. 


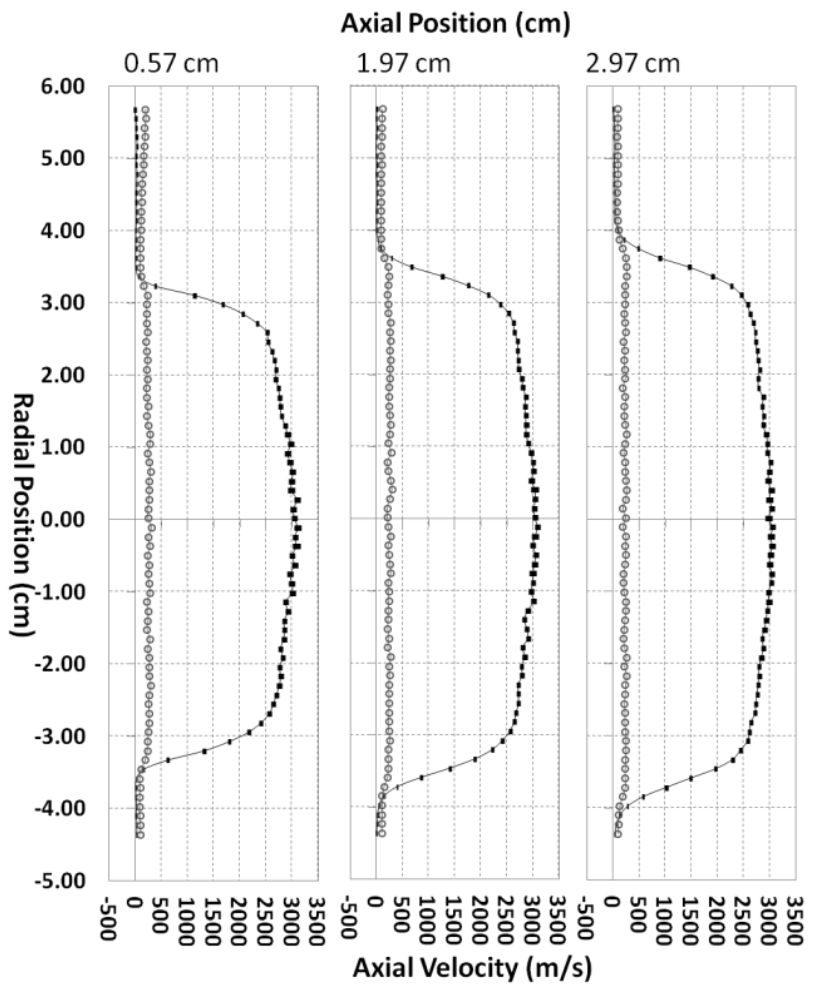

(a)

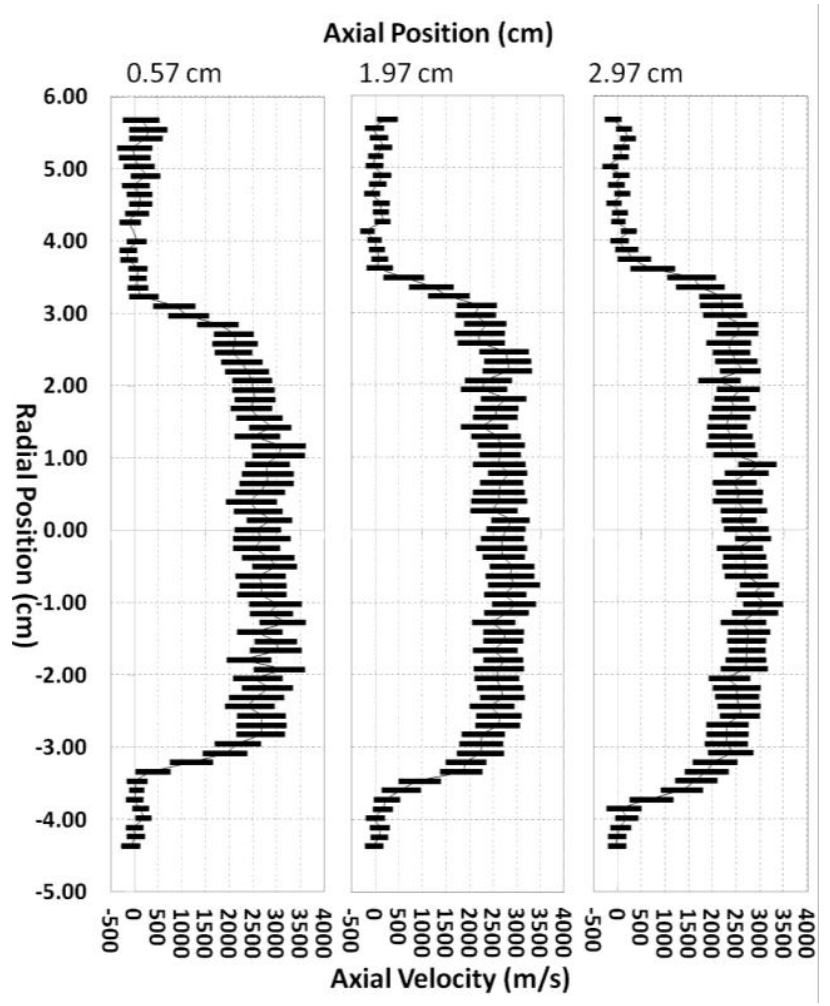

(b)

Figure 11. Axial velocity profiles obtained in the HYMETS facility: (a) mean (filled symbols) and fluctuating components (open symbols); (b) single-shot measurements. Width of bars indicates uncertainty in the measured value.

\section{Three-Laser $\mathrm{NO}_{2}$ Photolysis, NO Fluorescence MTV with a Double-Frame Camera}

In the previous section, a single-laser NO MTV measurement technique using a double-frame camera was described that was capable of providing both average and single-shot, single-component velocity profiles in a hypersonic flowfield. One of the primary motivations behind the development and application of the NO MTV technique was to make quantitative measurements of velocity in NASA hypersonic wind tunnel facilities in hypersonic boundary layers undergoing laminar-to-turbulent transition. However, in facilities such as the 31-inch Mach 10 blowdown tunnel at Langley, the higher Reynolds numbers required to achieve laminar-to-turbulent transition on a flat plate model requires that the facility stagnation pressure, model angle-of-attack (AoA), or both be increased. Either of these changes results in higher post-shock static pressures which in turn proportionally reduced the fluorescence lifetime. For static pressures higher than approximately $800 \mathrm{~Pa}$, the reduction in NO fluorescence lifetime is such that the intensity decay follows the trend of the dashed line shown in Fig. 8(a). Consequently, the fluorescence intensity of the molecules initially tagged by the probe laser is undetectable in the second exposure.

This issue prompted application of an $\mathrm{NO}_{2}$-to-NO photolysis molecular tagging velocimetry technique for use in NASA's 31-inch Mach 10 air blowdown wind tunnel. The technique had been previously developed and demonstrated by several researchers, though never before in a large-scale hypersonic wind tunnel. ${ }^{413-16}$ Rather than $\mathrm{NO}$ being seeded into the flow, it is generated via photolysis of $\mathrm{NO}_{2}$, which has been seeded into the flowfield. The photolysis of $\mathrm{NO}_{2}$ is induced by a high-intensity UV laser pulse. By dividing this laser pulse into multiple lines, a spatial pattern of $\mathrm{NO}$ can be "written" onto a gas flowfield containing $\mathrm{NO}_{2}$. Shortly after the pattern of $\mathrm{NO}$ is written onto the gas, two sequential pulses by a probe laser are formed into laser sheets which are then used to generate fluorescence where there is NO. This fluorescence is captured using the same double-frame camera described in the previous section. The benefit of this technique is that the time delay between the first and second exposures is not limited by the lifetime of NO fluorescence. Rather, the lines of NO can be interrogated for as long as they remain within the field of view and are not overly dissipated by diffusion. References 17-19 provide more detail on the application of this technique at NASA Langley Research Center.

Prior to performing these experiments, some changes were made to the experiment based on lessons learned while performing the single-laser NO MTV technique, described in the previous section. Several additional issues arose over the course of testing using the $\mathrm{NO}_{2}$ photolysis technique which affected the data quality and yield. 
Reference 18 details attempts at improving the initial experimental setup (described in Ref. 17) meant to increase the data quality and yield. These issues are described in the following paragraphs.

The laser system used for these experiments consisted of two Nd:YAGpumped dye laser systems in which the dye output is sum-frequency mixed to generate tunable UV light near $226 \mathrm{~nm}$. A $355 \mathrm{~nm}$ third-harmonic beam from one Nd:YAG laser and the two $226 \mathrm{~nm}$ beams were used as pump beams and probe laser sheets, respectively. The temporal relationship of these three beams is illustrated in Fig. 12. In this figure, the $355 \mathrm{~nm}$ pump beam was used to write the pattern of $\mathrm{NO}$ onto the gas flowfield. The NO generation occurs

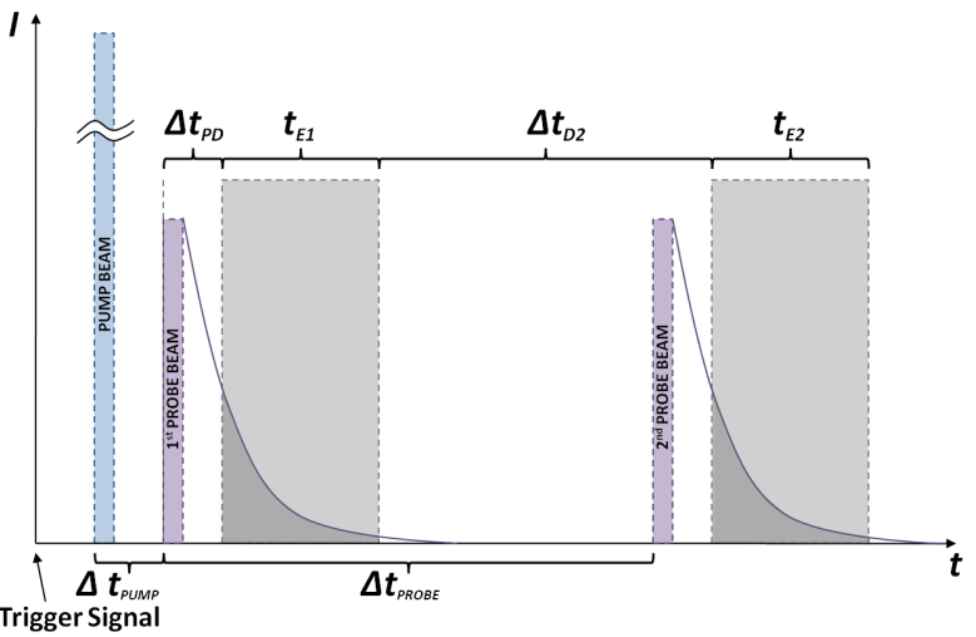
via photolysis of $\mathrm{NO}_{2}$, which was seeded

into the flow upstream of the measurement region. After a short delay period $\left(\Delta t_{\text {PUMP }}\right)$, the the first probe beam, which is formed into a laser sheet, generates fluorescence where there is NO. After a delay period corresponding to $\triangle t_{P R O B E}$ the second probe beam again generates fluorescence in the NO pattern, which has been displaced by the motion of the gas. The fluorescence signal, which decays exponentially with time, is captured using the same intensified double-frame camera described in section II.B. The sequential exposures, of duration $t_{E I}$ and $t_{E 2}$ respectively, are delayed from the probe beam excitation by $\Delta t_{P D}$ to minimize laser scatter off of the wind tunnel model surface.

Figure 13 provides a schematic of the optical components used to overlap the probe laser sheets with the pump beam. The view is from an upstream perspective looking downstream at the model. The exact spacing of the optics and the beam dimensions in this figure are not to scale.

In order to minimize undesirable laser scatter, the model was modified with a 50.8-mm outer diameter, 6.4-mm thick quartz window centered about the measurement region and mounted flush to the model surface. The use of this UV-transparent window was also intended to prevent damage to the model's black painted surface that would occur from the focused $355 \mathrm{~nm}$ pump beams. This modified model configuration is included in Fig. 13.

The two camera exposures, with gate durations of $t_{E 1}$ and $t_{E 2}$, were initially timed such that they completely enveloped the first and second probe beams, respectively. This was done to maximize the signal in either of the exposures; there was no longer the problem of having unequal intensities between the exposures resulting from fluorescence decay, as was a problem with the single-laser NO MTV technique. Unfortunately, scatter from the pump and probe beams off the bottom metallic surface of the model, observed on and through the quartz window insert, diminished the signal-to-noise ratio in both exposures. Inserting a second window into the model allowed light to pass out the bottom of the model, which further reduced laser scatter. Initially, two filters were used to block this scatter, one for the $355 \mathrm{~nm}$ light (Semrock HG01 $254 \mathrm{~nm}$ filter: <1\% transmission below $236 \mathrm{~nm}$ and above $263 \mathrm{~nm}$; >65\% from $244 \mathrm{~nm}-256$ $\mathrm{nm}$ ) and one for the $226 \mathrm{~nm}$ light (Layertec $\mathrm{GmbH}$ : <1\% transmission at $226 \mathrm{~nm}$; >80\% from $235 \mathrm{~nm}-280 \mathrm{~nm}$ ). However, it was determined that the $355 \mathrm{~nm}$ filter blocked approximately $75 \%$ of the NO fluorescence, thus drastically reducing signal levels. Therefore, a second approach was adopted using only the $226 \mathrm{~nm}$ filter. It consisted of delaying both camera exposures by approximately $\Delta t_{P D}=28 \mathrm{~ns}$ after the start of the respective probe laser pulses. This delay setting

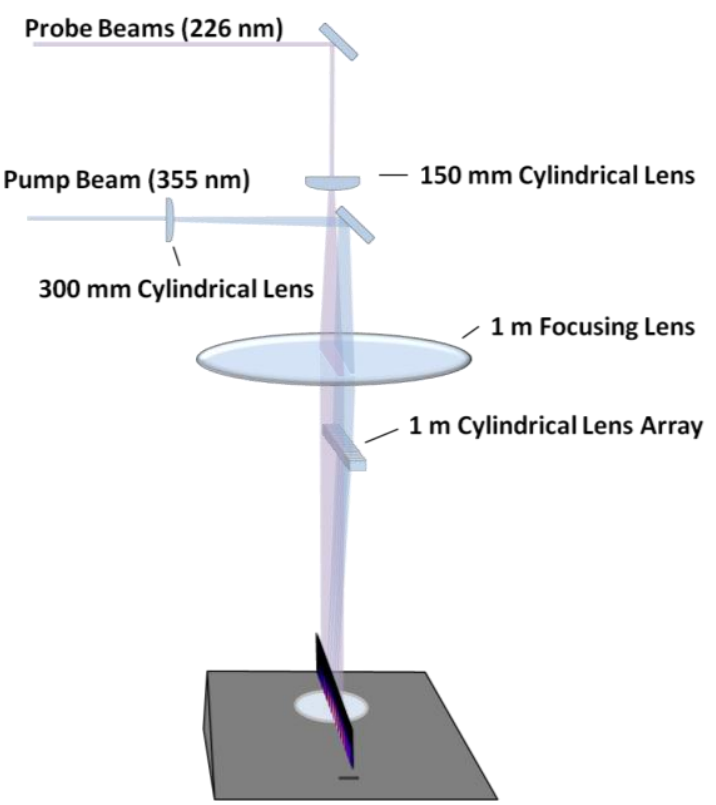

Figure 13. Schematic of laser alignment of three-laser $\mathrm{NO}_{2}$-to-NO photolysis experiments. (Taken from Ref. 18, Fig. 2)

12

American Institute of Aeronautics and Astronautics 
corresponded to a combined delay of $\Delta t_{P U M P}+\Delta t_{P D}=35.2 \mathrm{~ns}+28 \mathrm{~ns}=63.2 \mathrm{~ns}$ beyond the start of the pump laser. The collisional quenching at the conditions of interest (static pressure, $P_{\text {static }} \sim 3 \mathrm{kPa}$; edge temperature, $T_{e} \sim 240 \mathrm{~K}$ ) resulted in a relatively short NO fluorescence lifetime of approximately $30 \mathrm{~ns}-40 \mathrm{~ns}$. Although delaying the camera exposures reduced the amount of fluorescence signal collected, adequate signal-to-noise levels were achieved throughout the experiment. A feature of this timing arrangement was that the measurement became insensitive to timing jitter associated with the laser pulses (though sensitivity to timing jitter associated with the camera gates was retained).

Another improvement involved using a series of UV-reflective mirrors to guide both of the probe laser beams from the laser carts to the tunnel test section rather than anti-reflection coated $90^{\circ}$ turning prisms, as were used in the experiment described in Ref. 17. The turning prisms used in that experiment were measured to transmit only $70 \%-90 \%$ of the incident laser energy per prism. By using 99\% reflective dielectric turning mirrors, the laser energies for the pump, first probe, and second probe (measured at the test section prior to passing through the sheetforming and profile-forming optics) were approximately $10 \mathrm{~mJ} / \mathrm{pulse}, 3.1 \mathrm{~mJ} / \mathrm{pulse}$, and $4.3 \mathrm{~mJ} / \mathrm{pulse}$, respectively. In Ref. 17, these energies were approximately $11-14 \mathrm{~mJ} / \mathrm{pulse}, 0.7 \mathrm{~mJ} / \mathrm{pulse}$, and $0.7 \mathrm{~mJ} / \mathrm{pulse}$, respectively. This represented an improvement in the transmitted first and second probe laser energies by a factor of 4.4 and 6.1, respectively.

The pump beam alignment relative to the model surface was also modified. In Ref. 17, the angle of incidence was approximately $75^{\circ}$ with respect to the plate surface. This was done as a matter of convenience because plate angles of both $5^{\circ}$ and $20^{\circ}$ were being tested and hence the angle-of-incidence of the pump laser beams were aligned normal $\left(90^{\circ}\right)$ to the plate surface for the $5^{\circ}$ plate angle. At the $20^{\circ}$ plate angle, however, the pump beams were sloped in the streamwise direction with a $75^{\circ}$ angle-of-incidence relative to the plate surface. This made it difficult to analyze the data for time delays that were much greater than $1 \mu \mathrm{s}$ because a profile in the first exposure could potentially be overlapped by a neighboring profile in the second exposure. In Ref. 18 and 19, the angle of incidence was modified to be between approximately $87^{\circ}$ to $85^{\circ}$ with respect to the plate surface.

The magnification in Refs. 18 and 19 was 16.3 -pixels $/ \mathrm{mm}$, an improvement of approximately $8 \%$ from that in Ref. 17. This was accomplished by moving the camera closer to the test section. The horizontal spatial resolution of the measurement is determined by the width of a tagged profile $(0.6 \mathrm{~mm})$ and the total shift of the profile (which is largest at the maximum probe laser delay, $\Delta t_{P R O B E}=1 \mu \mathrm{s}$, and the maximum edge velocity of $1289 \mathrm{~m} / \mathrm{s}$ ). The resulting spatial resolution ranged from approximately $0.6 \mathrm{~mm}$ (where the velocity was low) to $1.3 \mathrm{~mm}$. If a $\triangle t_{P R O B E}$ $=2 \mu \mathrm{s}$ setting is used, the spatial resolution is reduced to $2.6 \mathrm{~mm}$. The vertical spatial resolution was approximately $0.08 \mathrm{~mm}$.

A study was performed in quiescent air to help determine the accuracy and precision of the technique. The circles in Fig. 14 show the computed average contribution of spatial uncertainties to the velocity uncertainty as a function of probe laser delay, $\Delta t_{P R O B E}$. The open symbols represent the current "best case" uncertainty for this technique when the SNR and data yield are relatively high. The filled symbols are more representative of typical SNR and data yields. This figure shows that the average uncertainty decreases monotonically with $\triangle t_{P R O B E}$ and follows the power fit relation provided in the upper righthand corner of the plot.

The squares in Fig. 14 are the mean velocity magnitude as a function of $\triangle t_{P R O B E}$, measured in a nominally static gas. For a truly static gas, these measurements quantify the systematic error associated with this velocity measurement technique. Again, the open symbols represent the "best case" SNR and data yield levels, while the filled symbols are more representative of SNR and data yield levels typically measured in the boundary

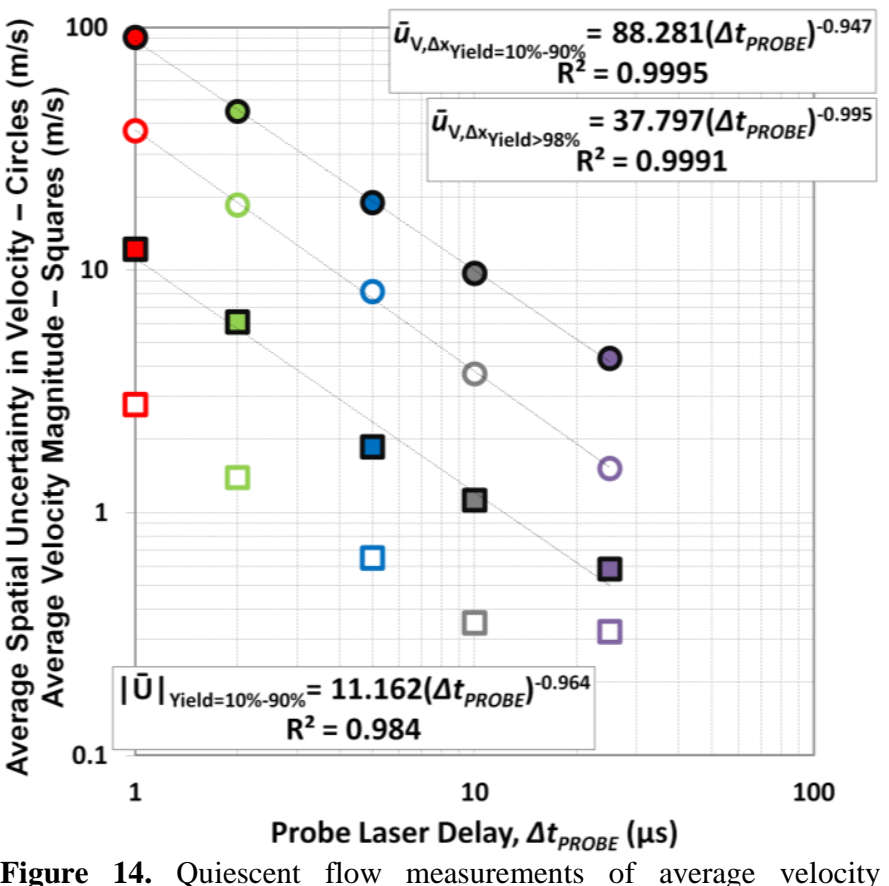

Figure 14. Quiescent flow measurements of average velocity magnitude (squares) and the associated average spatial uncertainty in layers. The filled squares follow the power fit velocity (circles) for high (open) and lesser (filled) data yields as
shown in the bottom left-hand corner of the plot. functions of $\Delta t_{P R O B E}$. $P_{\text {static }}=2.9 \mathrm{kPa}$ (Ref. 18, Fig. 6). 
While this trend in the mean velocity is similar to that of the spatial uncertainty, the $\Delta t_{P R O B E}=25 \mu$ s data point shows no significant reduction in velocity magnitude from that measured with the next shorter probe delay of $\Delta t_{P R O B E}=10 \mu \mathrm{s}$. This suggests that the nearly quiescent flow actually had small fluctuations in velocity on the order of $0.3 \mathrm{~m} / \mathrm{s}$; any measured velocities above this level are attributable to systematic errors in the measurement system.

Figure 15 shows, from top to bottom, average raw images captured in a laminar hypersonic boundary layer using this technique for $\triangle t_{P R O B E}$ settings of $1 \mu$ s (average of 205 single-shot images), $2 \mu$ s (average of 205 single-shot images), and $5 \mu \mathrm{s}$ (average of 81 single-shot images), respectively. For the $5 \mu \mathrm{s}$ image, the rate at which $\mathrm{NO}_{2}$ was seeded into the flow had been reduced to $8.8 \mathrm{mg} / \mathrm{s}$ from the $15.6 \mathrm{mg} / \mathrm{s}$ supplied during acquisition of the $1 \mu \mathrm{s}$ and 2 $\mu$ s images. For the $1 \mu \mathrm{s}$ and $2 \mu \mathrm{s}$ images, clearly defined profiles are observed. For the $5 \mu$ s data, profiles are nearly indistinguishable from their neighboring profiles. For this particular experimental configuration, this represents an upper bound on the $\Delta t_{P R O B E}$ setting (and therefore $\Delta t_{D 2}$ setting) that could be used. This is a limitation that occurs when the distance between the lines of $\mathrm{NO}$ written onto the gas is comparable to the flow spatial scale, $U_{e} \cdot \Delta t_{D 2}$, where $U_{e}$ is the velocity characteristic of the boundary layer edge condition. Similar problems of this nature can occur in low pressure flows where the mass diffusion time scale is much less than that of $\Delta t_{D 2}$. However, increasing $\triangle t_{P R O B E}$ (and therefore $\Delta t_{D 2}$ ) generally reduces the measurement uncertainty. This tradeoff between the measurement uncertainty and the flow spatial scale must be considered when performing such experiments.

Figure 16, from Ref. 18, shows mean velocity profiles for $\triangle t_{P R O B E}$ settings of $1 \mu \mathrm{s}$ (red data) and $2 \mu \mathrm{s}$ (green data). In each case, the mean velocities were computed from 205 single-shot images. The data between approximately $0.05 \mathrm{~mm}$ and $0.60 \mathrm{~mm}$ above the model surface agree relatively well with analytic solutions corresponding to each acquisition period. Near the wall, the velocity profiles do not tend linearly towards zero as expected (no-slip velocity wall condition). This non-zero velocity measurement error was attributed to pixel blooming and magnification issues. To reduce this error, the magnification could be increased resulting in an improved spatial resolution, allowing for a better resolved velocity profile, especially near the plate surface. Above $0.6 \mathrm{~mm}$, the mean velocity profiles in Fig. 16 are biased away from the analytic solution, with smaller than expected velocities relative to the analytic solutions. Several reasons for this discrepancy are discussed in Ref. 18, however it is thought that the method by which $\mathrm{NO}_{2}$ gas was seeded into the boundary layer flow likely had some influence.

Using this three-laser $\mathrm{NO}_{2}$-to-NO photolysis MTV technique with a double-frame camera, uncertainties in the mean velocity in a laminar boundary layer were as low as approximately $1 \%-2 \%$ the measured estimated edge velocity $(1289 \mathrm{~m} / \mathrm{s}) .{ }^{18}$ Single-shot uncertainties were, at best, approximately $5 \%$ of the edge velocity. By using a quartz window to minimize the laser scatter on the plate surface, measurements of streamwise velocity as close as $0.08-\mathrm{mm}$ to the plate surface were made in Refs. 18 and 19, representing a factor of 4 improvement compared to results in Ref. 7 using the single-laser NO MTV technique. 


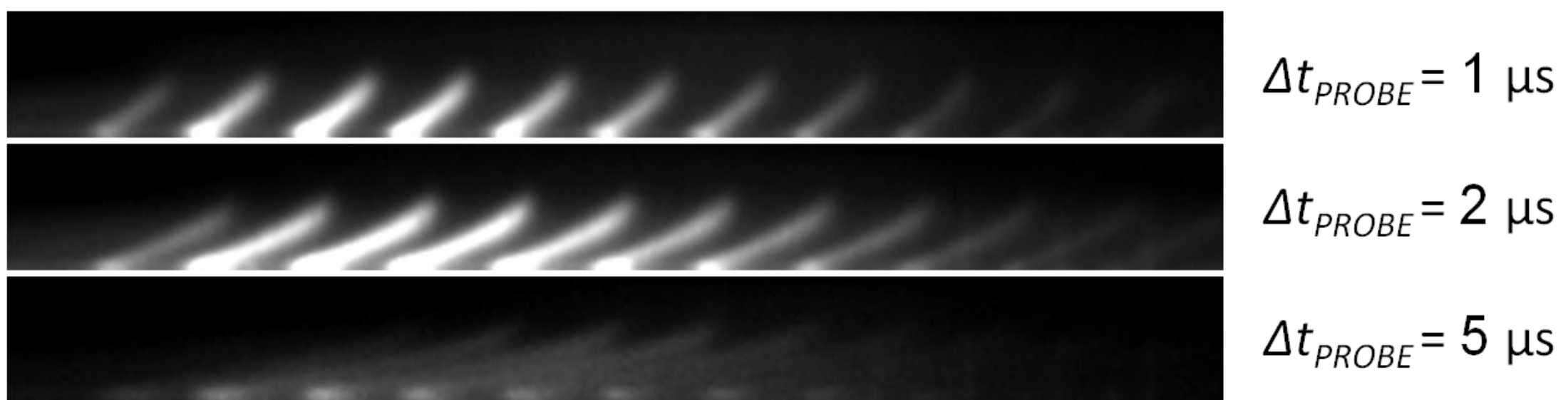

Figure 15. Average of raw images with $\triangle t_{P R O B E}$ settings of $1 \mu$ s (top, average of 205 images), $2 \mu$ s (middle, average of 205 images), and $5 \mu$ s (bottom, average of 81 images) (Taken from Ref. 18, Fig. 9).

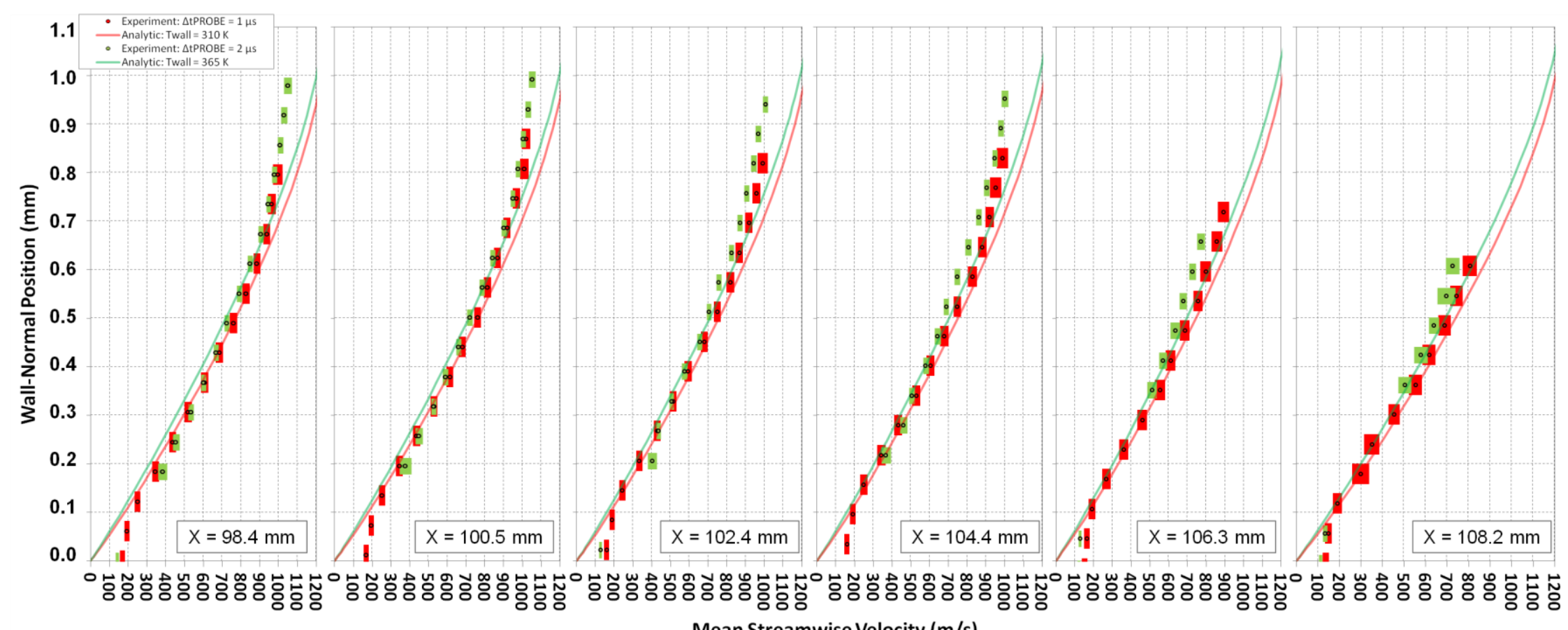

Mean Streamwise Velocity $(\mathrm{m} / \mathrm{s})$

Figure 16. Mean velocity profiles for $\triangle t_{P R O B E}$ settings of $1 \mu \mathrm{s}$ (red data points) and $2 \mu \mathrm{s}$ (green data points). Solid red and green lines correspond to analytic velocity boundary layer solutions for wall temperatures of $310 \mathrm{~K}$ and $365 \mathrm{~K}$, respectively (Taken from Ref. 18, Fig. 8). 


\section{Single-Laser Fluorescence-Based Doppler Velocimetry}

\section{Doppler Velocimetry Using Two-Photon Excitation of Oxygen Atom Fluorescence at a Point}

The HYMETS facility at NASA Langley is capable of achieving relatively high-enthalpy freestream conditions (ranging from approximately $5.3-26.8 \mathrm{MJ} / \mathrm{kg}$ ) and can simulate multiple environments consisting of varying concentrations of nitrogen $\left(\mathrm{N}_{2}\right)$, oxygen $\left(\mathrm{O}_{2}\right)$, carbon dioxide $\left(\mathrm{CO}_{2}\right)$, and argon $(\mathrm{Ar}){ }^{20}$ Quantifying the compositions, thermodynamic states, and velocities of the gases generated in this facility is important as these parameters play critical roles in materials research and development, such as those materials associated with thermal protection systems. Of particular interest is the behavior of atomic oxygen because of its chemical reactivity. These factors motivated a set of experiments in HYMETS, performed from October 2011 through January 2012, in which velocity measurements of atomic oxygen were performed using a Doppler-based laser-induced fluorescence velocimetry technique. The purpose of the measurements was to characterize facility operation.

Figure 17(a) shows a top-view schematic of the HYMETS facility with forward and return laser beams needed to perform oxygen atom (O-atom) LIF Doppler shift based velocimetry. The facility was run with air at 100 SLPM, $100 \mathrm{~A}$, and $15 \mathrm{MJ} / \mathrm{kg}$. Short duration pulses of $225.65 \mathrm{~nm}$ light were produced by an Nd:YAG-pumped dye laser (operating with Rhodamine 640 dye) and mixing crystal and were guided through one of the viewing ports of the HYMETS facility. This forward beam passed through the test section and exited a rear-facing viewing port into an optical delay line. The beam was then redirected back through the rear-facing viewing port and into the test section so that the forward and return beams were collinear. Lenses outside of both windows to focus the light to a small point in front of the test sample. The return beam passed back through an aperture located near the laser cart to ensure overlap of the beams at the same measurement location. A photomultiplier tube (PMT) collected the infrared O-atom LIF signal through a viewing port at the bottom of the facility (not shown in the figure). The use of the delay line allowed the signal from the forward beam to be temporally separated from the return signal. This temporal separation was much shorter than the flow time scale; in other words, the flow was virtually stationary between the two pulses.

During the experiments, the laser was scanned spectrally from $225.648 \mathrm{~nm}$ to $225.662 \mathrm{~nm}$, exciting three closely-spaced $\mathrm{O}$-atom transitions. The scan took approximately 1 minute to complete. These two-photon transitions included the excited states $\mathrm{J}^{\prime}=0,2$, and 1 from the $\mathrm{J}^{\prime \prime}=2$ ground state and are denoted as $\lambda_{1}, \lambda_{2}$, and $\lambda_{3}$, respectively, in Fig. 18. The spacing of these transitions was determined from the online NIST Atomic Spectra Database.*

Figure 17(b) shows a zoomed in view of the measurement region in which both the forward and return beams are collinear, passing ahead of the bow shock generated by the sample and crossing the axis of symmetry of the nozzle flow at their foci. Along the axis of symmetry, it is assumed that any radial component of velocity is negligible and that only an axial velocity component, $U$, exists. The component of axial velocity in the beam direction is $U_{b e a m}=$ $U \cdot \cos 49.7^{\circ}$. From the oxygen-atom frame of reference, the incident laser radiation at the focal point of the forward

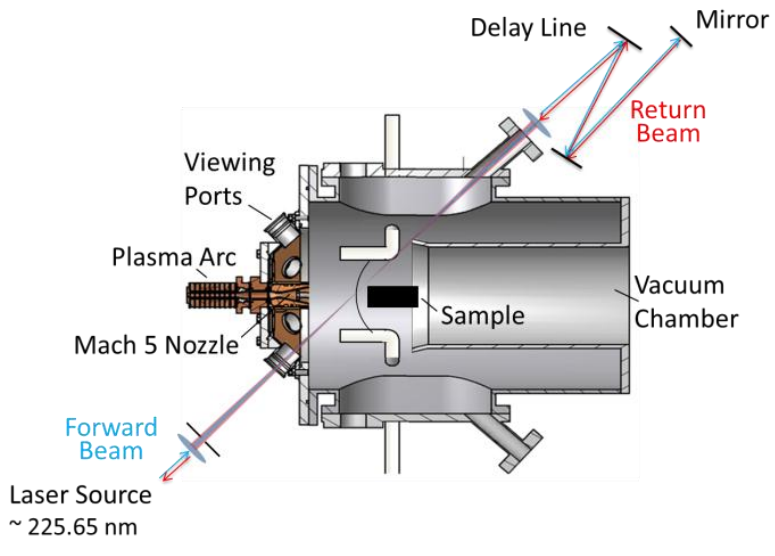

(a)

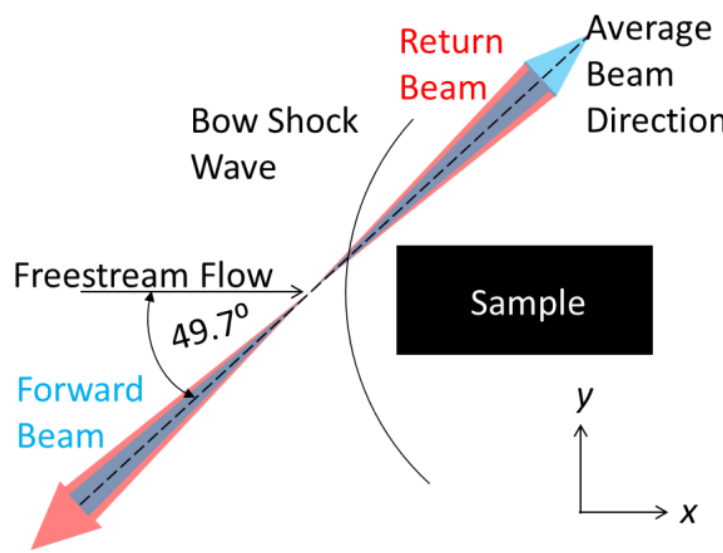

(b)

Figure 17. (a) HYMETS facility and O-atom LIF Doppler shift velocimetry experimental setup; (b) Zoom in of sample showing laser beam direction relative to sample and freestream flow. (adapted from Ref. 10, Fig. 1)

\footnotetext{
* Access to NIST Atomic Spectra Database available at: http://www.nist.gov/pml/data/asd.cfm [data retrieved October 30, 2012]
} 
and return beams appears either red- or blue-shifted, respectively. As a result, the spectral frequency at which the laser radiation from the forward and return beams is absorbed is blue- and red-shifted, respectively, from the laboratory frame of reference. This is realized experimentally by observing the respective shifted $\mathrm{O}$-atom LIF signals measured with the PMT.

Figure 18 shows the excitation spectrum of the three blue-shifted (BS) transitions $\left(\lambda_{1, \mathrm{BS}}, \lambda_{2, \mathrm{BS}}\right.$, and $\left.\lambda_{3, \mathrm{BS}}\right)$ and red-shifted (RS) transitions $\left(\lambda_{1, \mathrm{RS}}, \lambda_{2, \mathrm{RS}}\right.$, and $\left.\lambda_{3, \mathrm{RS}}\right)$ as measured with the PMT, which correspond to the excitation by the forward and return beams, respectively. The spectral positions of these transitions are governed by the following relationship:

$$
U=\frac{c}{\cos 49.7^{\circ}} \frac{\Delta v_{\text {Doppler Shift }}}{2 \cdot v_{\text {Laser }}}=\frac{c}{\cos 49.7^{\circ}} \frac{\left(v_{B S}-v_{R S}\right)}{\left(v_{B S}+v_{R S}\right)}
$$

where $c$ is the speed of light and the wavelength and wave number are related by:

$$
v\left[\mathrm{~cm}^{-1}\right]=\frac{10^{7}\left[\mathrm{~cm}^{-1} \cdot \mathrm{nm}\right]}{\lambda[\mathrm{nm}]}
$$

The primary motivation of using this counter-propagating, collinear laser beam arrangement is that a reference signal indicating the center frequency of the laser, $v_{\text {Laser }}$, is not required. This is because both the blue and red Doppler shifts are of equal but opposite magnitude according to $-\Delta v_{\text {Doppler Shift } \quad \text { and }}$ $+\Delta v_{\text {Doppler Shift, }}$ respectively. As such, the measured frequency shift between the blue- and redshifted LIF signals is equal to twice the magnitude of the Doppler shift, $\Delta v_{\text {Doppler Shift }}$, measured between the respective blue- $\left(v_{\mathrm{BS}}\right)$ and red-shifted $\left(\nu_{\mathrm{RS}}\right)$ frequency signals and the laser frequency $\left(v_{\text {Laser }}\right)$.

Based on the results shown

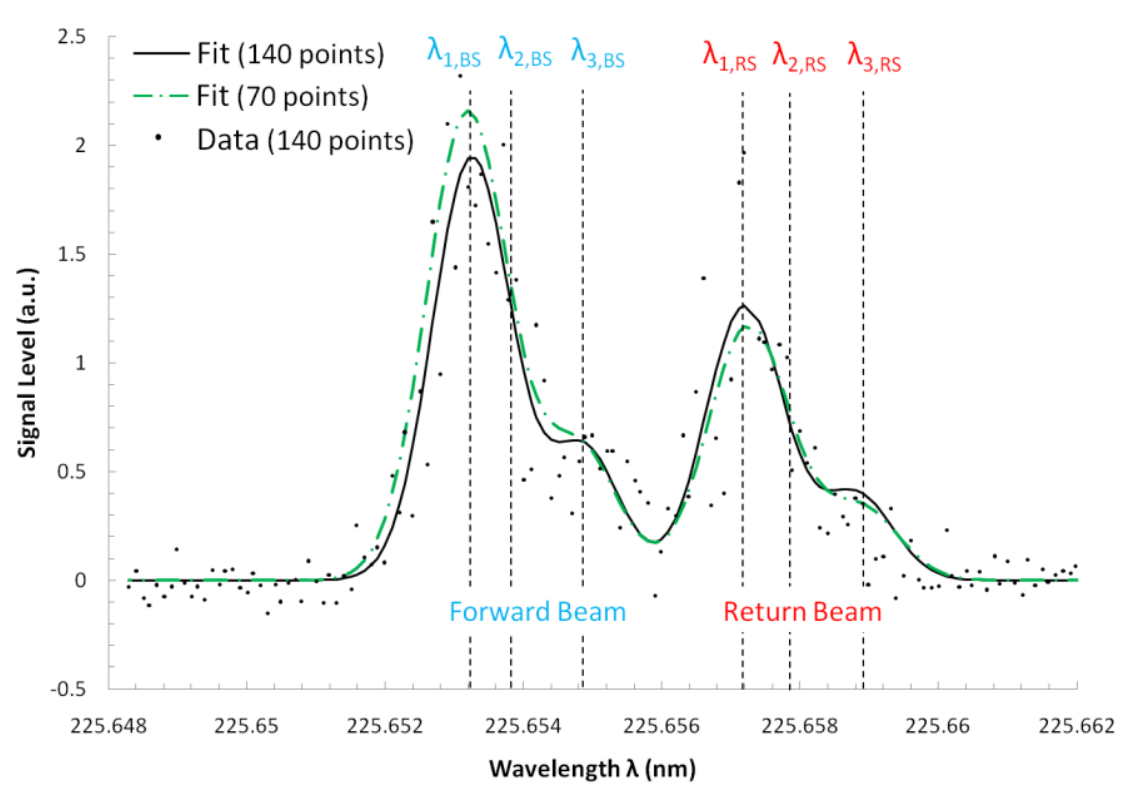

Figure 18. Raw O-atom LIF signal (points) with overlaid red-shifted (RS) and blueshifted (BS) curve fits (lines). in Fig. 18, a least-squares of the error between the experimental data and Gaussian curve fits gives a Doppler shift of approximately $0.002 \mathrm{~nm}$ in each direction. This corresponds to a velocity of $U_{\text {beam }}=2620 \mathrm{~m} / \mathrm{s}$ in the direction of the forward beam and $U=$ $4060 \mathrm{~m} / \mathrm{s}$ in the axial direction. The green dash-dot line in Fig. 18 represents a curve fit to one half of the PMT data points (70) in order to estimate the error of the measurement technique. Based on the comparison between the curve fits, there is an estimated error of $\pm 120 \mathrm{~m} / \mathrm{s}$ in the axial velocity measurement although further analysis is needed to fully quantify this error.

\section{Doppler Velocimetry Using NO Fluorescence in a Plane}

Using the same Doppler shift principle described in the previous section, measurements of radial velocity across a plane were also performed in the HYMETS facility using NO LIF. The measurements, detailed in Ref. 10, were intended to provide an improved understanding of the operation of that arcjet's flowfield characteristics. In the measurements, the spectral position of the laser was scanned over a small wavelength range about the excitation spectrum of the relatively well-isolated $\mathrm{Q}_{11}(13)$ transition of NO.

Figure 19 shows a schematic of the HYMETS facility and the laser alignment used for the radial velocity measurements. The laser beam in the experiment was formed into a thin sheet using a method similar to that shown in Fig. 1 and directed into the HYMETS facility via a viewing port fitted with a quartz window. A periscope (two 
mirrors, indicated by thick black lines in Fig. 19) inside the test chamber directed the laser sheet to the flow. The resulting fluorescence was captured in a series of images using a Princeton Instruments PIMAX-2 intensified CCD camera with 512 x 512 pixel array.

For this analysis, the laser scanning rate was synchronized to the camera system which allowed for the fluorescence signal (which varied over the acquisition period) to be related to the laser's wavelength. The images were then analyzed with the post-processing software, ImageJ, to establish a temporal distribution of the fluorescence signal extracted from the image sequence at each spatial location.

Sources of systematic error in the measurement included wavelength-scanning nonlinearities of the laser, collisional shifts in the absorption transition, and laser beam attenuation in the radial direction resulting from absorption by NO. The error due to wavelength-scanning nonlinearity arises from discrepancies between the wavelength

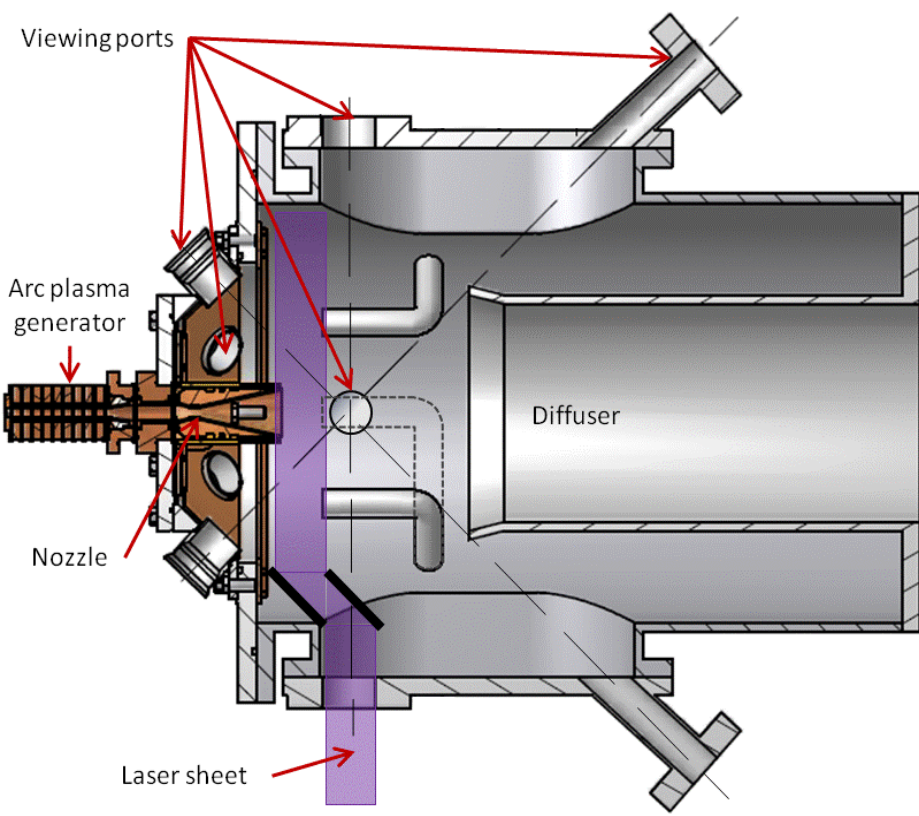

Figure 19. Schematic of the HYMETS test section and with laser alignment used for radial NO LIF Doppler velocimetry (Ref. 10, Fig. 1).

indicated by the software controlling the laser and the actual wavelength. Using scan linearity measurements obtained from the manufacturer, a worst case error of approximately $\pm 0.0045 \mathrm{~cm}^{-1}$ in $\Delta v_{\text {Doppler Shift }}$ per wave number scanned was calculated. This would lead to a systematic error in the measured radial velocity of approximately $\pm 0.9 \%$. Thus, the maximum error in velocity due to non-linearity of the laser scanning was $\pm 4.5 \mathrm{~m} / \mathrm{s}$.

Although the flow in the HYMETS facility is chemically reacting - and so a perfect gas analysis does not strictly apply - a perfect gas assumption was used for estimating static conditions in the jet in order to approximately quantify the magnitude of the collisional shift and its influence on measurement uncertainty. By assuming an isentropically expanded Mach 5 jet having a stagnation pressure (arc pressure) of $1.1 \mathrm{~atm}$, the pressure shift at the nozzle exit was computed to be approximately $0.0016 \mathrm{~cm}^{-1}$. This corresponded to a systematic error of just $1 \mathrm{~m} / \mathrm{s}$ due to the neglect of collisional broadening. This error was partially mitigated through the method by which the laser's center wavelength, $\lambda_{\mathrm{L}}$, was calculated. Since $\lambda_{\mathrm{L}}$ was calculated from analysis of regions in the flow without a radial velocity component, any pressure shift resulting from a large uniform pressure field would be accounted for.

The process of laser attenuation by NO absorption, described by Beer's law of absorption, explains the approximately exponential decrease in laser intensity observed in the experiment as the laser passed through the flowfield. This was a source of error because it artificially amplifies $\Delta v_{\text {Doppler Shift }}$ in regions of high absorption and large radial velocity. For example, in a diverging flow like the one being studied, the gas that sees the laser most blue-shifted is also the gas that sees the greatest laser intensity, and so would yield the largest fluorescence signal. This biased weighting would look like a spectral shift in the data. Consequently, an over prediction in the magnitude of radial velocity occurs. To correct for this error, a laser intensity profile including the effects of absorption was simulated. First, the absorption coefficient was assumed to be constant along the path of the laser sheet. By imposing symmetry on the fluorescence intensity profile, it was possible to calculate a radial profile of laser intensity and fit it with an exponential function modeling the laser intensity with

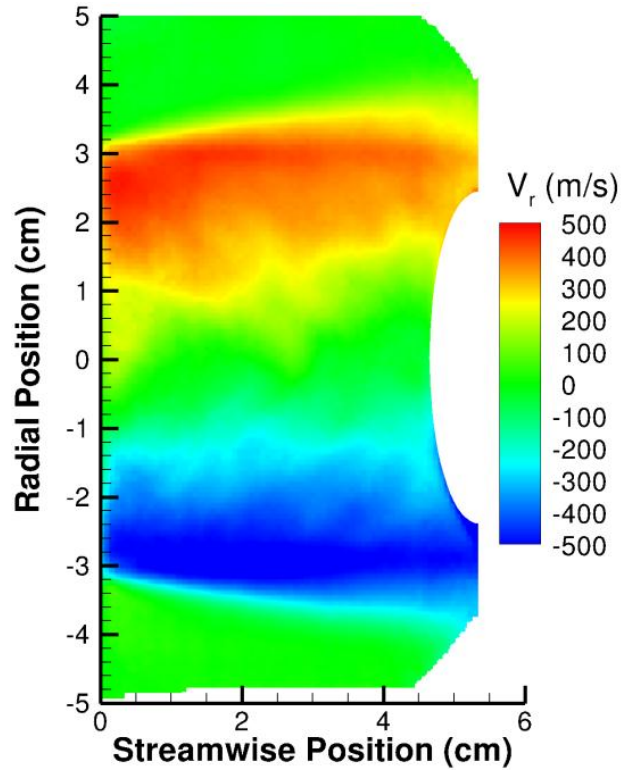

Figure 20. Comparison of radial distribution of velocity measured in HYMETS shown as a contour plot for $6.5 \mathrm{MJ} / \mathrm{kg}$ condition simulating Earth's atmosphere (adapted from Ref. 10, Fig. 20). 
absorption. After applying this simulated laser intensity profile to the experimental data and re-fitting the shifted spectra, the systematic error in the Doppler-shifted absorption peaks, $\lambda_{c}$, was calculated. For the experiment, the maximum uncertainty due to laser beam attenuation was estimated to be $\pm 56 \mathrm{~m} / \mathrm{s}$.

Figure 20 shows a contour map of the velocity measured in HYMETS for a $6.5 \mathrm{MJ} / \mathrm{kg}$ operating condition simulating Earth's atmosphere. In this measurement, the magnitude of radial velocity in the top portion of the image (positive radial velocity) was found to be approximately 25\% larger than that measured in the lower portion of the image. This suggests that the systematic error in radial velocity attributed to laser beam attenuation may not be completely described by the constant absorption coefficient assumption used to quantify this error. Further study is needed to completely understand this effect.

\section{Conclusions}

This paper summarizes high-speed velocity measurements performed at NASA Langley Research Center using laser-induced fluorescence-based velocimetry methods. The measurement techniques discussed included variants of the molecular tagging velocimetry (MTV) and fluorescence-based Doppler velocimetry techniques. These measurements were performed in a variety of high-speed test facilities and applied to several different flowfields. The paper provided details on the setup of each experiment, difficulties encountered while performing the measurements, improvements made to each setup in response to the difficulties, and sample results from each measurement.

\section{Acknowledgments}

The authors wish to thank the facility operators in NASA Langley's 15-Inch Mach 6, 31-Inch Mach 10, and HYMETS facilities, as well as the many collaborators who contributed to this work. This work was supported primarily by the NASA Aeronautics Research Mission Directorate's Fundamental Aeronautics Program, Hypersonics Project.

\section{References}

1 Inman, J.A., Danehy, P.M., Bathel, B.F., Alderfer, D.W., and Novak, R.J., "Laser-Induced Fluorescence Velocity Measurements in Supersonic Underexpanded Impinging Jets," $48^{\text {th }}$ AIAA Aerospace Sciences Meeting, AIAA Paper 20101438, January 4-7, 2010, Orlando, FL.

2 Inman, Jennifer A., "Fluorescence Imaging Study of Free and Impinging Supersonic Jets: Jet Structure and Turbulent Transition," Ph.D. Dissertation, Department of Physics, The College of William and Mary, Williamsburg, VA, 2007

${ }^{3}$ Wilkes, Jennifer A., Christopher E. Glass, Paul M. Danehy, and Robert J. Nowak, "Fluorescence Imaging of Underexpanded Jets and Comparison with CFD," AIAA-2006-0910, 44 ${ }^{\text {th }}$ AIAA Aerospace Sciences Meeting and Exhibit, Reno, NV, 9-12 January 2006.

${ }^{4}$ Orlemann, C., Schulz, C., and Wolfrum, J., "NO-flow tagging by photodissociation of NO2. A new approach for measuring small-scale flow structures," Chem. Phys. Lett., 307, pp. 15-20, 1999.

${ }^{5}$ Bathel, B.F., Danehy, P.M., Inman, J.A., Jones, S.B., Ivey, C.B., and Goyne, C.P., "Multiple Velocity Profile Measurements in Hypersonic Flows Using Sequentially-Imaged Fluorescence Tagging," $48^{\text {th }}$ AIAA Aerospace Sciences Meeting, AIAA Paper 2010-1404, January 4-7, 2010, Orlando, FL.

${ }^{6}$ Bathel, B.F., Danehy, P.M., Inman, J.A., Jones, S.B., Ivey, C.B., and Goyne, C.P., "Velocity Profile Measurements in Hypersonic Flows Using Sequentially-Imaged Fluorescence-Based Molecular Tagging Velocimetry,” AIAA Journal, Vol. 49, No. 9, September 2011. doi: 10.2514/1.J050722

${ }^{7}$ Bathel, B.F., Danehy, P.M., Inman, J.A., Watkins, A.N., Jones, S.B., Lipford, W.E., Goodman, K.Z., Ivey, C.B., and Goyne, C.P., "Hypersonic Laminar Boundary Layer Velocimetry with Discrete Roughness on a Flat Plate," AIAA Paper 2010-4998, 40th Fluid Dynamics Conference and Exhibit, Chicago, IL, June 28 - July 1, 2010.

8 Johansen, C.T., Danehy, P.M., Ashcraft, S.W., Bathel, B.F., Inman, J.A., and Jones, S.B., "PLIF study of Mars Science Laboratory capsule reaction control system jets," $41^{\text {st }}$ AIAA Fluid Dynamics Conference, AIAA Paper 2011-3890, June 2730, 2011, Honolulu, HI.

9 Johansen, C.T., Novak, J., Bathel, B., Ashcraft, S.W., and Danehy, P.M., "Comparison of MSL RCS jet computations with flow visualization and velocimetry," 50 ${ }^{\text {th }}$ AIAA Aerospace Sciences Meeting, AIAA Paper 2012-0594, January 4-7, 2012, Nashville, TN.

${ }^{10}$ Inman, J.A., Bathel, B.F., Johansen, C.T., Danehy, P.M., Jones, S.B., Gragg, J.G., and Splinter, S.C., "Nitric Oxide PLIF Measurements in the Hypersonic Materials Environmental Test System (HYMETS)," $49^{\text {th }}$ AIAA Aerospace Sciences Meeting, AIAA Paper 2011-1090, Orlando, FL, January 4-7, 2011. 
${ }^{11}$ Danehy, P.M., O’Byrne, S., Houwing, A.F.P, Fox, J.S., and Smith, D.R., “Flow-Tagging Velocimetry for Hypersonic Flows Using Fluorescence of Nitric Oxide," AIAA Journal, 41 (2), pp. 263-271, February, 2003. doi: 10.2514/2.1939

12 Iyer, P., Muppidi, S., and Mahesh, K., "Roughness-Induced Transition in High Speed Flows," 49 ${ }^{\text {th }}$ AIAA Aerospace Sciences Meeting, AIAA Paper 2011-566, Orlando, FL, January 4-7, 2011.

${ }^{13}$ Shinji, N., Kasahara, M., Tsue, M., and Kono, M., "Velocity Measurements of Reactive and Non-reactive Flows by NO-LIF Method Using $\mathrm{NO}_{2}$ Photodissociation," Heat Transfer - Asian Research, 34 (1), pp. 40-52, 2005. doi: $10.1002 / \mathrm{htj} .20038$

${ }^{14}$ Hsu, A.G., Srinivasan, R., Bowersox, R.D.W., and North, S.W., "Two-component molecular tagging velocimetry utilizing NO fluorescence lifetime and NO2 photodissociation techniques in an underexpanded jet flowfield," Applied Optics, 48 (22), pp. 4414-4423, August, 2009.

${ }^{15}$ Hsu, A.G., Srinivasan, R., Bowersox, R.D.W, and North, S.W., “Molecular Tagging Using Vibrationally Excited Nitric Oxide in an Underexpanded Jet Flowfield,” AIAA Journal, 47 (11), pp. 2597-2604, November, 2009. doi: $10.2514 / 1.47716$

${ }^{16}$ Jiang, N., Nishihara, M., and Lempert, W.R., "500 kHz $\mathrm{NO}_{2}$ Molecular Tagging Velocimetry in a Mach 5 Wind Tunnel," AIAA 2010-4348, $27^{\text {th }}$ AIAA Aerodynamic Measurement Technology and Ground Testing Conference, Chicago, IL, June 28 - July $1,2010$.

17 Bathel, B.F., Johansen, C.T., Danehy, P.M., Inman, J.A., Jones, S.B., and Goyne, C.P., "Hypersonic Boundary Layer Transition Measurements Using $\mathrm{NO}_{2} \rightarrow \mathrm{NO}$ Photo-dissociation Tagging Velocimetry," AIAA Paper 2011-3246, 41 ${ }^{\text {st }}$ AIAA Fluid Dynamics Conference and Exhibit, June 27-30, 2011, Honolulu, HI.

${ }^{18}$ Bathel, B.F., Danehy, P.M., Johansen, C.T., Jones, S.B., and Goyne, C.P., "Hypersonic Boundary Layer Measurements with Variable Blowing Rates Using Molecular Tagging Velocimetry," AIAA Paper 2012-2886, $28^{\text {th }}$ AIAA Aerodynamic Measurement Technology, Ground Testing, and Flight Testing Conference, June 25-28, 2012, New Orleans, LA.

19 Bathel, B.F., Johansen, C.T., Jones, S.B., Danehy, P.M., and Goyne, C.P., "Trip-induced Transition Measurements in a Hypersonic Boundary Layer Using Molecular Tagging Velocimetry," To be presented at the $51^{\text {st }}$ AIAA Aerospace Sciences Meeting, January 7-10, 2013, Grapevine, TX.

${ }^{20}$ Splinter, S.C., Bey, K.S., and Gragg, J.G., "Comparative Measurements of Earth and Martian Entry Environments in the NASA Langley HYMETS Facility," AIAA Paper 2011-1014, 49 ${ }^{\text {th }}$ AIAA Aerospace Sciences Meeting, January 4-7, 2011, Orlando, FL. 